\title{
Benchmark models, planes, lines and points for future SUSY searches at the LHC
}

\author{
S.S. AbdusSalam ${ }^{1}$, B.C. Allanach ${ }^{2}$, H.K. Dreiner ${ }^{3}$, J. Ellis ${ }^{4,5}$, U. Ellwanger ${ }^{6}$, J. Gunion ${ }^{7}$, S. Heinemeyer ${ }^{8}$, \\ M. Krämer ${ }^{9}$, M. Mangano ${ }^{5, \text { a }}$, K.A. Olive ${ }^{10}$, S. Rogerson ${ }^{11}$, L. Roszkowski ${ }^{12,13}$, M. Schlaffer ${ }^{14,15}$, G. Weiglein $^{15}$ \\ ${ }^{1}$ The Abdus Salam International Centre for Theoretical Physics, Strada Costiera 11, 34014 Trieste, Italy \\ ${ }^{2}$ Department of Applied Mathematics and Theoretical Physics, Cambridge University, Wilberforce Road, Cambridge CB3 0WA, UK \\ ${ }^{3}$ Bethe Center for Theoretical Physics \& Physikalisches Institut der Universität Bonn, Bonn, Germany \\ ${ }^{4}$ Theoretical Particle Physics and Cosmology Group, Department of Physics, King's College London, London WC2R 2LS, UK \\ ${ }^{5}$ CERN, 1211 Genève 23, Switzerland \\ ${ }^{6}$ Laboratoire de Physique Théorique, UMR 8627, CNRS and Université de Paris-Sud, Bât. 210, 91405 Orsay, France \\ ${ }^{7}$ Department of Physics, University of California, Davis, CA 95616, USA \\ ${ }^{8}$ Instituto de Física de Cantabria (CSIC-UC), 39005 Santander, Spain \\ ${ }^{9}$ Institute for Theoretical Particle Physics and Cosmology, RWTH Aachen University, 52056 Aachen, Germany \\ ${ }^{10}$ William I. Fine Theoretical Physics Institute \& Department of Physics, University of Minnesota, Minneapolis, MN 55455, USA \\ ${ }^{11}$ High Energy Physics Group, Blackett Laboratory, Imperial College, Prince Consort Road, London SW7 2AZ, UK \\ ${ }^{12}$ National Centre for Nuclear Research, Hoza 69, 00-681 Warsaw, Poland \\ ${ }^{13}$ Department of Physics and Astronomy, University of Sheffield, Sheffield S3 7RH, UK \\ ${ }^{14}$ Arnold Sommerfeld Center for Theoretical Physics, Ludwig-Maximilians-Universität München, Fakultät für Physik, 80333 Munich, Germany \\ ${ }^{15}$ DESY, Notkestraße 85, 22607 Hamburg, Germany
}

Received: 4 November 2011 / Published online: 17 December 2011

(C) The Author(s) 2011. This article is published with open access at Springerlink.com

\begin{abstract}
We define benchmark models for SUSY searches at the LHC, including the CMSSM, NUHM, mGMSB, mAMSB, MM-AMSB and p19MSSM, as well as models with R-parity violation and the NMSSM. Within the parameter spaces of these models, we propose benchmark subspaces, including planes, lines and points along them. The planes may be useful for presenting results of the experimental searches in different SUSY scenarios, while the specific benchmark points may serve for more detailed detector performance tests and comparisons. We also describe algorithms for defining suitable benchmark points along the proposed lines in the parameter spaces, and we define a few benchmark points motivated by recent fits to existing experimental data.
\end{abstract}

\section{Introduction}

Softly broken low-energy supersymmetry (SUSY) has many attractive features [1-4]. For example, unlike the Standard Model (SM), it provides an elegant mechanism for stabilizing the gauge hierarchy with respect to the effects of radiative corrections and a natural weakly-interacting dark mat-

a e-mail: michelangelo.mangano@cern.ch ter (DM) candidate, in addition to facilitating gauge coupling unification, predicting the existence of a light Higgs boson and potentially making a desirable contribution to the anomalous magnetic moment of the muon, $(g-2)_{\mu}$. On the other hand, SUSY has to be (softly) broken in order to make contact with reality, which even in the general Minimal Supersymmetric Standard Model (MSSM) introduces a large number of new free parameters, such as the soft SUSYbreaking (SSB) masses, mixings and complex phases in the couplings. Because of SUSY's natural link with grand unified theories (GUTs), one often explores SUSY models in which various boundary conditions are imposed on the SSB parameters at the GUT scale.

ATLAS and CMS have already made great strides in searches for SUSY with $\sim 1 \mathrm{fb}^{-1}$ of data each [5-23]. However, the presentation of the experimental results from searches for supersymmetric particles or other kinds of new physics at the LHC necessarily involves a certain dependence on the assumed model of new physics. Results from SUSY searches are usually presented either within the parameter space of a specific SUSY model or as cross section limits or limits on the masses of particles of "simplified models" [24]. While the latter approach provides in principle information that is less model-dependent, the former approach has the advantage that the specific SUSY models can be confronted also with other constraints, such as lim- 
its from Higgs searches and searches for dark matter as well as constraints from electroweak precision data and flavour physics.

In order to allow the interpretation of the experimental results in terms of different possible manifestations of SUSY it is useful to consider certain benchmark models that have fewer free parameters than the most general incarnation of the MSSM. For this purpose we consider several models with GUT-scale boundary conditions on the SSB parameters that have frequently been studied up to now in the context of collider and dark matter searches. As an alternative, we also consider a version of the MSSM with 19 parameters, as well as models with R-parity violation and the NMSSM. Within those models we propose some benchmark subspaces that could be useful for the presentation of experimental results. We also define specific benchmark points that illustrate different possible experimental signatures and may serve for more detailed detector performance tests and comparisons. We choose those benchmark points along certain lines in the parameter spaces that share distinctive experimental signatures, which may differ from those in the models most commonly studied to date. If the SUSY exclusion limits advance, one can move to the next benchmark point along any of these lines. This could be useful for investigating the prospective experimental sensitivity or for optimising the search in the relevant parameter region, etc.

\section{Definition of models}

\subsection{The Constrained MSSM (CMSSM)}

A commonly studied model within this broad class of GUTbased models is the Constrained MSSM (CMSSM) [25-40], in which not only gaugino soft masses unify to a common value $m_{1 / 2}$ at the GUT scale, but also the SSB masses of all the sfermions and Higgs doublets unify to a common value $m_{0}$. These parameters, along with a common tri-linear SSB parameter $A_{0}$, the ratio of Higgs vacuum expectation values $\tan \beta$ and the sign of the Higgs mixing parameter, $\operatorname{sign}(\mu)$, form the four continuous and one discrete parameters of the model:

CMSSM: $\quad m_{1 / 2}, m_{0}, A_{0}, \tan \beta, \operatorname{sign}(\mu)$.

The unified parameters $m_{1 / 2}, m_{0}, A_{0}$ are thought to appear via some gravity-mediated mechanism, and are defined at the GUT scale $M_{\mathrm{GUT}} \approx 2 \times 10^{16} \mathrm{GeV}$, whereas $\tan \beta$ (and $\operatorname{sign}(\mu)$ ) are defined at the electroweak (EW) scale. The CMSSM is assumed to conserve multiplicatively the discrete R-parity, $R_{P} \equiv(-\mathbf{1})^{2 S+L+3 B}$, where $S$ is the spin of the particle, $L$ the lepton number and $B$ the baryon number. This ensures that the lightest supersymmetric particle (LSP) is stable and a natural cold dark matter (CDM) candidate, often thought to be the lightest neutralino. The CMSSM, like many other simple unified models, is a Minimal Flavor-Violating (MFV) scenario with no additional flavor-violating terms beyond those in the SM.

\subsection{The minimal supergravity model (mSUGRA)}

Additional assumptions may be imposed beyond those in the CMSSM. For example, in the minimal supergravity model (mSUGRA) there is a specific relation between the trilinear and bilinear SSB parameters and the universal scalar mass: $A_{0}=B_{0}+m_{0}{ }^{1}$ and the gravitino mass $m_{3 / 2}$ is fixed to be equal to the common scalar mass before renormalization, $m_{3 / 2}=m_{0}[42-46]$. Hence, the model has just three free continuous parameters,

mSUGRA: $m_{1 / 2}, m_{0}, A_{0}, \operatorname{sign}(\mu)$,

and $\tan \beta$ is now fixed by the radiative electroweak symmetry breaking conditions.

Relaxing the condition on the gravitino mass leads to the Very Constrained MSSM (VCMSSM) [47, 48], which has the same set of free parameters as mSUGRA but may evade the restrictive cosmological and astrophysical constraints due to late decays of neutralinos into gravitinos. ${ }^{2}$

\subsection{Non-universal Higgs mass model (NUHM)}

Because of its economy, the CMSSM (and a fortiori more restricted versions) may be missing some features of unified models with less restrictive boundary conditions at the unification scale. In particular, the assumption of unification of the SSB Higgs mass with those of the sfermions may easily be relaxed without prejudice to MFV [49-56]. Depending on whether the two soft SSB parameters of the Higgs sector are the same (NUHM1) or not (NUHM2, often simply termed the NUHM) the free parameters of this model are

NUHM1: $m_{1 / 2}, m_{0}, m_{H}, A_{0}, \tan \beta, \operatorname{sign}(\mu)$,

where $m_{H}$ denotes the unified SSB parameter in the Higgs sector at $M_{\mathrm{GUT}}$, or

NUHM2: $\quad m_{1 / 2}, m_{0}, m_{H_{u}}, m_{H_{d}}, A_{0}, \tan \beta, \operatorname{sign}(\mu)$,

where $m_{H_{u}}$ and $m_{H_{d}}$ denote the two independent soft SSB parameters in the Higgs sector at $M_{\mathrm{GUT}}$.

\footnotetext{
${ }^{1}$ It should be noted that many publicly available codes use a different sign convention, $A_{0}=B_{0}-m_{0}$, as may be ascertained by comparing the signs of the gauge and Yukawa contributions to the renormalization group equations of the $A$ parameters. More details can be found in [41].

${ }^{2}$ The conditions (2) are often applied at the unification scale $M_{\mathrm{GUT}}$, though the underlying theory may enforce them at some other scale such as the Planck scale, and renormalization of the SSB parameters between this scale and $M_{\mathrm{GUT}}$ could be significant.
} 
Equivalently, one can trade the new GUT scale parameters for one or two parameters at the EW scale. In the NUHM1, $m_{H}$ can be traded for $M_{A}$ or $\mu$, whereas in the NUHM2 both $m_{H_{u}}$ and $m_{H_{d}}$ can be traded for $M_{A}$ and $\mu$ as free parameters.

\subsection{Minimal gauge-mediated SUSY breaking (mGMSB)}

The gauge-mediated SUSY-breaking (GMSB) model is constructed from a GUT-scale scenario for SUSY-breaking mediation in which communication to the visible sector is via gauge interactions [57, 58]. Minimal GMSB (mGMSB) models have four continuous parameters, namely the messenger field mass-scale, $M_{\text {mess }}$, the visible-sector SSB scale, $\Lambda, \tan \beta, c_{\text {grav }} \geq 1$ (a factor for the gravitino mass) as well as a discrete parameter, $N_{\text {mess }}$, representing the number of $S U(5)$ representations of the mediating fields:

mGMSB: $\quad M_{\text {mess }}, \Lambda, \tan \beta, c_{\text {grav }}, N_{\text {mess }}$.

The next-to-lightest supersymmetric particle (NLSP) plays an important role in the phenomenology, since cascade decay chains of each produced sparticle will typically end in the NLSP. It is commonly the stau, which decays to a tau plus gravitino, or a neutralino, which often decays to a photon plus a gravitino. Depending on the parameters, the NLSPs can range between being stable on the time-scales taken to cross a detector, to being prompt and decaying at the interaction point. For example, in the case of a neutralino NLSP, its decay length is approximately given by [61]

$$
\begin{aligned}
L_{\text {decay }}= & \frac{1}{\kappa_{\gamma}}\left(\frac{100 \mathrm{GeV}}{m_{\mathrm{NLSP}}}\right)^{5}\left(\frac{\Lambda}{100 \mathrm{TeV}}\right)^{2}\left(\frac{M_{\text {mess }}}{100 \mathrm{TeV}}\right)^{2} \\
& \times 10^{-4} \mathrm{~m},
\end{aligned}
$$

where $\kappa_{\gamma}$ is the photino component of the neutralino. Here $\Lambda$ sets the sparticle mass scale, and varying $M_{\text {mess }}$ changes the decay length according to (6). We include in our list of proposed benchmarks below a model with a quasi-stable neutralino NLSP. According to (6), this can be arranged by increasing $M_{\text {mess }}$. If $M_{\text {mess }}$ is close to the GUT scale, the resulting spectra can closely resemble those of the CMSSM [62], but an intermediate $M_{\text {mess }}$ can yield spectra that significantly differ from typical CMSSM ones. Some similar features may be found in models that assume universal SSB parameters at a scale below the GUT scale [63-65].

\subsection{Anomaly-mediated SUSY breaking (AMSB)}

The anomaly-mediated SUSY-breaking (AMSB) model is constructed from a GUT-scale scenario for SUSY-breaking mediation in which communication to the visible sector arises from the super-Weyl anomaly [59, 60]. In minimal
AMSB (mAMSB) models there are three parameters: a parameter, $m_{0}$, contributing to the squared scalar masses at the GUT scale which would otherwise be negative; the vacuum expectation value of the supergravity field representing the sparticle mass scale, $m_{\text {aux }}$, and $\tan \beta$ :

mAMSB: $m_{0}, m_{\text {aux }}, \tan \beta$.

2.6 Mixed modulus-anomaly-mediated SUSY breaking (MM-AMSB)

Mixed modulus-anomaly-mediated SUSY breaking scenarios are inspired by models of string compactification with fluxes [66]. In these scenarios the soft terms receive contributions from both gravity-mediated and anomaly-mediated SUSY breaking [67], whose relative sizes are characterized by a phenomenological parameter $\alpha$. This mixed scenario is also known as "mirage mediation", since it appears that the gaugino masses unify at an intermediate scale well below $M_{\mathrm{GUT}}$. MM-AMSB models are specified by the parameters

MM-AMSB: $m_{3 / 2}, \alpha, \tan \beta, \operatorname{sign}(\mu), n_{i}, l_{a}$,

where $\alpha$ determines the relative weight of anomaly and gravity mediation, the $n_{i}$ are the modular weights of the visible sector matter fields, and $l_{a}$ appears in the gauge kinetic function [68]. In MM-AMSB models the relative size of the gaugino masses $M_{1}, M_{2}$ and $M_{3}$ is determined by the parameter $\alpha$, and different values of $\alpha$ thus correspond to different mass patterns for the strongly- and weakly-interacting gauginos.

\subsection{The phenomenological 19-parameter MSSM} (p19MSSM)

A complementary framework to the above GUT-scale models for SUSY breaking is the phenomenological MSSM, with all of its many free parameters specified at the electroweak scale. In order to make a phenomenological analysis of its vast parameter space manageable, various wellmotivated simplifying assumptions have been made. On the other hand, some of the main assumptions used in the previous sections in reducing the number of MSSM parameters to construct the GUT-scale models, namely, the physics behind SUSY breaking, the mediation mechanism and the renormalization group (RG) running of the parameters from the SSB scale are not relevant for, and hence decoupled from, typical MSSM parametrizations.

One MSSM parametrization that has been recently extensively studied involves 19 free parameters of the MSSM (p19MSSM) [69-72]. In this approach, the resulting number of SSB parameters are derived from the parent 105 parameters of the MSSM by removing all sources of CP violation and generation mixing beyond the SM: assuming the parameters to be real, that all off-diagonal elements in the sfermion mass matrices are equal to zero, and that first- and second- 
generation soft terms are equal. Only the trilinear couplings most relevant for SUSY effects, $A_{t}, A_{b}, A_{\tau}$ are included as free parameters. The other $A_{f \neq t, b, \tau}$ are set to zero.

The free parameters of the p19MSSM model are

p19MSSM:

$M_{1}, M_{2}, M_{3}$;

$m_{\tilde{u}_{L}}=m_{\tilde{d}_{L}}=m_{\tilde{c}_{L}}=m_{\tilde{s}_{L}}, \quad m_{\tilde{u}_{R}}=m_{\tilde{c}_{R}}$,

$m_{\tilde{d}_{R}}=m_{\tilde{s}_{R}}, \quad m_{\tilde{e}_{L}}=m_{\tilde{\mu}_{L}}, \quad m_{\tilde{e}_{R}}=m_{\tilde{\mu}_{R}} ;$

$m_{\tilde{t}_{L}}=m_{\tilde{b}_{L}}, m_{\tilde{t}_{R}}, m_{\tilde{b}_{R}}, m_{\tilde{\tau}_{L}}, m_{\tilde{\tau}_{R}}$

$A_{t}, A_{b}, A_{\tau} ; \quad \mu, M_{A}, \tan \beta$,

where $M_{1,2,3}$ are the SSB parameters in the gaugino and gluino sector, $m_{\tilde{f}_{L}}$ and $m_{\tilde{f}_{R}}$ are the diagonal SSB parameters in the sfermion sector ( $f=u, d, c, s, t, b, e, \mu$, or $\tau$ ), and $A_{f=t, b, \tau}$ denote the trilinear Higgs-sfermion coupling in the third generation. An alternative choice of parameterisation would be to replace the CP-odd neutral Higgs mass parameter, $M_{A}$, and the Higgs doublet mixing parameter, $|\mu|$, by the two SSB parameters in the Higgs sector, $m_{H_{u}}^{2}$ and $m_{H_{d}}^{2}$.

Other versions of the MSSM with different choices of simplifying assumptions, leading to either a reduced or enlarged number of free parameters, have also been extensively studied in the literature. For some recent work done in the context of the LHC, see, e.g. [62, 73-75, 119].

\subsection{MSSM with RPV}

\subsubsection{Definition of the RPV-CMSSM}

As stated above, in the CMSSM R-parity $R_{p}$ is assumed to hold. Initially, the justification for this symmetry was that it guarantees the stability of the proton, and another attractive feature is that it leads to a good dark matter candidate such as the lightest neutralino.

In the RPV-MSSM the discrete symmetry R-parity is replaced by a different symmetry (such as baryon-triality, or lepton-parity), which guarantees proton stability but allows for either lepton-number violation or baryon-number violation. The LSP is then not stable, and can decay inside or outside the detector, depending on the size of the coupling. Another particle such as the axion, axino or gravitino [76] must then comprise the dark matter in the universe. On the other side, the advantage is that the neutrino masses are automatically included and naturally light [77].

The extra possible couplings in the superpotential are

$$
\begin{aligned}
W_{\mathrm{RPV}}= & \epsilon_{a b}\left[\frac{1}{2} \lambda_{i j k} L_{i}^{a} L_{j}^{b} E_{k}^{C}+\lambda_{i j k}^{\prime} L_{i}^{a} Q_{j}^{b} D_{k}^{C}+\kappa_{i} L_{i}^{a} H_{2}^{b}\right] \\
& +\frac{1}{2} \epsilon_{x y z} \lambda_{i j k}^{\prime \prime} U_{i}^{x C} D_{j}^{y C} D_{k}^{z C},
\end{aligned}
$$

where $i, j, k \in\{1,2,3\}$ are generation indices, $a, b \in\{1,2\}$ and $x, y, z \in\{1,2,3\}$ are $\mathrm{SU}(2)$ and $\mathrm{SU}(3)$ fundamental representation indices, respectively, and ${ }^{C}$ is the usual charge-conjugation. The parameters of the constrained RPV-MSSM (RPV-CMSSM; corresponding to the CMSSM) are [77]

RPV-CMSSM: $m_{1 / 2}, m_{0}, A_{0}, \tan \beta, \operatorname{sign}(\mu), \Lambda$,

where the CMSSM parameters are all defined at the unification scale, and $\Lambda$ corresponds to exactly one of the R-parity violating parameters

$\Lambda \in\left\{\lambda_{i j k}, \lambda_{i j k}^{\prime}, \lambda_{i j k}^{\prime \prime}, \kappa_{i}\right\}$

Note that in the CMSSM version discussed in [77], $\Lambda$ was defined at $M_{\mathrm{GUT}}$, whereas here we define it at $M_{Z}$, in order to make the analysis of the possible topologies easier. For the bilinear terms $\kappa_{i} L_{i} H_{u}, \kappa_{i}\left(M_{\mathrm{GUT}}\right)=0$ at the unification scale. There are however other models that have just one bilinear term, non-zero at the unification scale: $\Lambda=\kappa_{i} \neq 0$ : see for example [78]. Note furthermore, that even though just one RPV-coupling is non-zero at the unification scale in these models, the RGEs generate other non-zero couplings at the weak scale.

Due to the diverse phenomenology that may arise from the RPV couplings, we summarize here its general features. ${ }^{3}$

\subsubsection{Phenomenology of the RPV MSSM}

In the RPV MSSM the LSP typically decays promptly in the detector for couplings larger than about $10^{-6}$. The resulting number of different possible new signals coming from the RPV MSSM is large, since there are 48 new couplings, see (10). Furthermore, one can obtain single sparticle production, unlike in the R-parity conserving case, if one assumes that $\lambda_{i j k}^{\prime}$ or $\lambda_{\ell, m, n}^{\prime \prime}$ is at least larger than about $3 \times 10^{-3}$, for first-generation incoming quarks $(j, k=1$; $\ell, m=1$ or $m, n=1$ ) and even larger otherwise. For many values of $i, j, k$, large RPV couplings are strongly bounded from above by existing low-energy data, depending upon sparticle masses. We therefore concentrate here on the small RPV coupling case, where there should be no contradiction with experimental RPV bounds over regions of MSSM parameter space that are viable in the R-parity conserving limit.

We now list some possible signatures. With small RPV couplings, one tends to have the usual two-sparticle production via gauge couplings, in particular strong production of

\footnotetext{
${ }^{3}$ The RPV-CMSSM as described is programmed in SOFTSUSY, with the SUSY Les Houches accord option for inputting $\Lambda\left(M_{Z}\right)$ BLOCK SOFTSUSY parameter 8 , as described in the SOFTSUSY manual [79, 80].
} 
gluinos and squarks. These go through the usual cascade decays until the LSP is reached, which decays via the RPV coupling into Standard Model particles. Note, however, that the LSP need not be the neutralino. For small RPV couplings there are also large regions of parameter space where the stau is the LSP. This is for small $m_{0}$ and large $m_{1 / 2}$. These regions also exist in the R-parity conserving CMSSM, but are not considered on cosmological grounds. For a neutralino LSP, the experimental topologies are as those in the R-parity conserving limit, but instead of missing transverse momentum, additional leptons and jets are obtained from the LSP decay. For a stau LSP there are typically additional tau leptons in the final state. Whatever the LSP, if the RPV coupling is very small, much less than $10^{-6}$, one gets delayed LSP decays, leading to displaced vertices, and for the stau a potentially charged track.

We shall first consider how the RPV couplings change the R-parity conserving topologies in the case with a neutralino LSP and strong sparticle production. The minimal case is di-squark production, where each squark decays to a jet and a neutralino, which then decays via the RPV coupling. Producing gluinos, which then decay via squarks, can add additional jets, and there may be other particles in the chain leading to the additional emission of leptons, weak gauge bosons or jets. These features suggest inclusive searches, where one allows these particles in addition to the ones listed. Since the cascades are usually decaying on-shell particles, one can examine distributions of invariant masses of the final state particles in order to reconstruct bumps over the backgrounds. There is no expectation of flavour democracy among the couplings, so flavour subtraction may be used if backgrounds must be beaten down.

For $\lambda_{i j k}$ couplings, a neutralino LSP decays into two charged leptons and a neutrino. Thus the inclusive minimal signature is two jets, two leptons of one flavour, two leptons of another flavour (which may be the same flavour as the first pair) and a moderate amount of intrinsic missing energy. The leptons may or may not be taus for each case.

For $\lambda_{i j k}^{\prime}$ couplings, a neutralino LSP decays to a lepton (approximately half the time into a charged lepton and half the time into a neutrino which yields a small amount of missing transverse momentum) plus two jets. Thus, the inclusive signal is 4 jets and two leptons. The lepton may be either a tau or electron or muon.

In the case of $\lambda_{i j k}^{\prime \prime}$ couplings, a neutralino LSP decays to three jets via a virtual squark. In the case that $i, j$ or $k$ are 3 , the jets could be $t$ or $b$ quarks. Thus, the difficult minimal inclusive signal in this case is (from di-squark production, where each squark decays to a jet and a neutralino LSP) eight jets with no intrinsic missing transverse momentum. As mentioned above, two of the jets may be $b$ jets, and two may be replaced by decaying tops.

Next, we consider the stau-LSP case. Again the production is dominated by squark and/or gluino pair production.
The cascade decay will typically go via a virtual lightest neutralino, which is usually the NLSP. This then decays to a tau and the stau LSP. In the case that $L_{3}$ is involved with the RPV superpotential coupling directly, then the stau LSP decays directly into two particles as follows: in the case of $\lambda_{3 j k}^{\prime}$ it decays into two jets, whereas in $\lambda_{i j k}$ where $i$ or $k=3$, it decays into a lepton and a neutrino. Thus, these topologies are covered by just assuming a neutralino LSP and using the signature given above in the $\lambda_{i j k}^{\prime}$ or $\lambda_{i j k}$ paragraphs.

Finally, we discuss the case that $L_{3}$ does not appear in the RPV coupling. In this case, the stau decays through a fourbody decay [77] into a tau and whatever decay products the neutralino would decay to, as specified above. For $\lambda_{i j k}$, the stau will decay to a tau, two charged leptons and a neutrino. The inclusive signal is thus four charged leptons $(e, \mu)$, two taus and some missing transverse momentum. For $\lambda_{i j k}^{\prime}$ the stau decays to a tau and a lepton (neutral or charged) and two jets. The inclusive signal is therefore 4 jets, two leptons and two taus. For $\lambda_{i j k}^{\prime \prime}$ the stau will decay to a further tau plus 3 jets. The signature is thus as in the neutralino-LSP case ( 8 jets) supplemented by four taus. With good tau-id the additional taus could help search for the most difficult scenario. On the other hand, cases where one can obtain electrons or muons from the cascade decay chain are likely to be more feasible in terms of discovery.

\subsection{NMSSM}

The Next-to-Minimal Supersymmetric Standard Model (NMSSM) [81-84] is the simplest supersymmetric extension of the SM with a scale invariant superpotential: the $\mu$-term in the superpotential of the MSSM is replaced by a coupling to a gauge singlet superfield $S$, whose vev generates automatically an effective $\mu$-term of the order of the SUSY breaking scale. The superpotential of the NMSSM contains the terms

$W_{\mathrm{NMSSM}}=\lambda S H_{u} H_{d}+\frac{\kappa}{3} S^{3}$,

and the NMSSM specific soft terms are a soft mass $m_{S}^{2}$ for the scalar components of $S$ as well as trilinear couplings $\lambda A_{\lambda} S H_{u} H_{d}+\frac{\kappa}{3} A_{\kappa} S^{3}$. Just like the MSSM, the NMSSM solves the gauge hierarchy problem, provides a DM candidate, and leads to gauge coupling unification. The presence of $S$ implies an extended Higgs sector (3 neutral CP-even and 2 neutral CP-odd states) and an extended neutralino sector (5 neutralinos including the singlino). In cases where the LSP is singlino-like there will be significant modifications of all sparticle decay cascades as compared to the MSSM. The extended Higgs sector can include light CP-odd or CPeven states implying dominant Higgs-to-Higgs decays, and Higgs production in sparticle decay cascades.

Because of a larger number of both superpotential and soft SUSY breaking parameters than in the CMSSM, there 
are more options for selecting unified boundary conditions at the GUT scale. In particular, one can make less restrictive assumptions on the Higgs sector soft terms at the GUT scale [85]. Indeed, it is entirely possible that the soft terms for the singlet differ from those for the matter residing in complete SU(5) multiplets.

In the most constrained version, dubbed cNMSSM $[86,87]$, all soft terms are assumed to be universal at the GUT scale just as in the CMSSM. The cNMSSM has as many free parameters as the CMSSM: the parameters $\mu$ and $B$ are replaced by $\lambda$ and $\kappa$. With $\kappa$ being determined by $M_{Z}$, one is left with

cNMSSM: $m_{1 / 2}, m_{0}, A_{0}, \lambda, \operatorname{sign}(\mu)$.

However, phenomenological constraints imply that $m_{0}$ and $\lambda$ are small [86, 87]; with $A_{0}$ being determined by the DM relic density, one is left with $m_{1 / 2}$ as the only essential free parameter (as a function of which also $\tan \beta$ is determined $[86,87])$.

In the CNMSSM on the other hand one allows $m_{S}$ to differ from $m_{0}$ [88], in which case the free parameters are

CNMSSM: $m_{1 / 2}, m_{0}, A_{0}, \lambda, \tan \beta, \operatorname{sign}(\mu)$.

Even more relaxed boundary conditions in the Higgs sector have been considered. Leaving $A_{\kappa}, A_{\lambda}$ and $m_{S}$ as free parameters and allowing as well non-universality for the Higgs soft masses squared lead to a very much broader range of possible phenomenologies as compared to the cNMSSM. For example, it is easy to find fully consistent scenarios with a singlino-like LSP and light neutral Higgs bosons. Once $\kappa$ is determined by $M_{Z}$ and $m_{S}$ by $\tan \beta$, the parameter space of the semi-constrained SNMSSM can be taken as [85]

sNMSSM: $\lambda, m_{1 / 2}, m_{0}, m_{H_{u}}, m_{H_{d}}, A_{0}$,

$$
A_{\kappa}, A_{\lambda}, \tan \beta, \operatorname{sign}(\mu) .
$$

\section{Definition of benchmark planes, lines and points}

One may consider two distinct approaches to the specification of benchmarks for future SUSY searches at the LHC and elsewhere. One possible approach is based directly on the latest available experimental information. This has the advantage of incorporating all the relevant experimental constraints, but also the disadvantage of potentially becoming outmoded as the experimental constraints evolve. The other approach is to define specific benchmark scenarios that illustrate specific phenomenological possibilities, as exemplified by the SPS points and lines defined a decade ago [89]. These have the advantage that many applicable studies have already been made, but on the other hand these points and lines may incorporate the present experimental constraints only partially. For example, respect of the cosmological DM density constraint is not guaranteed, though one should bear in mind that there are alternative sources of DM, and that in some models, e.g., the RPV MSSM, the DM particle might not be found among the spartners of SM particles.

The most commonly used benchmark scenarios for early LHC searches were defined in [89], where lines and points in the CMSSM, mGMSB and mAMSB were specified, as well as in the CMSSM with non-universal $m_{1 / 2}$. Those Snowmass Points and Slopes (SPS) comprise six benchmark points (SPS 1a, SPS 1b, .., SPS 5) defined within the CMSSM, one CMSSM-like point with non-unified gaugino masses (SPS 6), two mGMSB points (SPS 7, SPS 8) and one mAMSB point (SPS 9). The status of those benchmark points with respect to the limits from early LHC SUSY searches is shown in Table 1. As indicated there, all the CMSSM points and the CMSSM-like point have now been excluded by early LHC data. On the other hand, the mGMSB points and the mAMSB point, which have a larger splitting between the coloured and the colour-neutral part of the spectrum, continue to be valid also in view of the limits from LHC SUSY searches with up to $\sim 2 \mathrm{fb}^{-1}$.

In this section we propose new benchmark planes, lines and points, guided also by global SUSY fits. In our proposal we keep the SPS benchmark points of the mGMSB and mAMSB scenarios, ${ }^{4}$ supplementing them with further points along the parameter lines. We propose updated parameter points and lines for the CMSSM, and we incorporate also further SUSY scenarios that were not considered in the SPS benchmarks. In addition, we extend our consideration of benchmark lines and points to the NMSSM and to RPV models, which, as described above, can allow for significantly different phenomenology as compared to the MSSM benchmarks. Overall, by broadening the model basis, the experimental results obtained by ATLAS and CMS can be expressed in a more general way, and their application to a wider class of models would be feasible. Input files for the generation of Monte Carlo events for each of the proposed benchmark points, in the standard Les Houches accord format [90], are available upon request.

\subsection{Current SUSY fits}

Several groups have published SUSY fits to the available data, both before and after incorporation of the published 2010 LHC data [91, 96-109], some fits have been made [110-112] incorporating the preliminary CMS and ATLAS results based on $\sim 1 \mathrm{fb}^{-1}$ of data, and attempts have also been made to foresee the evolution of these fits if the

\footnotetext{
${ }^{4} \mathrm{We}$ adopt here the definition in terms of the GUT-scale input parameters, while the original definition in [89] was based on the weak-scale parameters.
} 
Table 1 List of the SPS benchmark points, with their status with respect to the current limits from LHC SUSY searches, adapted from [91]. For each point the columns labelled A, B, C and D give the cross section for each of the signal regions used in the 35/pb ATLAS 0-lepton analysis [10]. The last column shows which SPS points were excluded by the absence of a signal in one or more of these four signal regions, or by the results of a subsequent ATLAS 0-lepton analysis using 165/pb of 2011 data [92]. "Allowed" reflects the lack of constraints from currently available results of searches with up to $\sim 1 \mathrm{fb}^{-1}$. In the mGMSB scenario the NLSP was taken to be stable on collider time scales. The starred cross sections are computed at leading order, whereas all the other values are calculated at next-to-leading order (NLO) [93-95]

\begin{tabular}{|c|c|c|c|c|c|c|}
\hline \multirow[t]{2}{*}{ Benchmark point } & \multirow[t]{3}{*}{ Model scenario } & \multicolumn{4}{|l|}{$\sigma / \mathrm{pb}$} & \multirow{3}{*}{$\frac{\text { Status }}{\text { ATLAS } 35,165 / \mathrm{pb}}$} \\
\hline & & $\overline{\mathrm{A}}$ & $\mathrm{B}$ & $\mathrm{C}$ & $\mathrm{D}$ & \\
\hline ATLAS Limits & & 1.3 & 0.35 & 1.1 & 0.11 & \\
\hline SPS 1a [89] & CMSSM & 2.031 & 0.933 & 1.731 & 0.418 & $\mathrm{~A}, \mathrm{~B}, \mathrm{C}, \mathrm{D}$ \\
\hline SPS 1b [89] & CMSSM & 0.120 & 0.089 & 0.098 & 0.067 & $165 / \mathrm{pb}$ \\
\hline SPS 2 [89] & CMSSM & 0.674 & 0.388 & 0.584 & 0.243 & $\mathrm{~B}, \mathrm{D}$ \\
\hline SPS 3 [89] & CMSSM & 0.123 & 0.093 & 0.097 & 0.067 & $165 / \mathrm{pb}$ \\
\hline SPS 4 [89] & CMSSM & 0.334 & 0.199 & 0.309 & 0.144 & $\mathrm{D}$ \\
\hline SPS 5 [89] & CMSSM & 0.606 & 0.328 & 0.541 & 0.190 & $\mathrm{D}$ \\
\hline SPS 6 [89] & CMSSM (non-universal $m_{1 / 2}$ ) & 0.721 & 0.416 & 0.584 & 0.226 & $\mathrm{~B}, \mathrm{D}$ \\
\hline SPS 7 [89] & mGMSB ( $\left.\tilde{\tau}_{1} \mathrm{NLSP}\right)$ & 0.022 & 0.016 & 0.023 & 0.015 & allowed \\
\hline SPS 8 [89] & $\operatorname{mGMSB}\left(\tilde{\chi}_{1}^{0} \mathrm{NLSP}\right)$ & 0.021 & 0.011 & 0.022 & 0.009 & allowed \\
\hline SPS 9 [89] & mAMSB & $0.019^{*}$ & $0.004^{*}$ & $0.006^{*}$ & $0.002^{*}$ & allowed \\
\hline
\end{tabular}

LHC does not discover SUSY with varying amounts of integrated luminosity [97]. These fits are based on a variety of statistical approaches, both Bayesian and frequentist, and the agreement between them gives some hope for the stability and reliability of the results.

Figure 1 displays the results of some recent CMSSM SUSY fits including $(g-2)_{\mu}$, in the $\left(m_{0}, m_{1 / 2}\right)$ plane (above) and the $\left(\tan \beta, m_{1 / 2}\right)$ plane (below). The best-fit points for different data sets are indicated by different symbols: closed stars for pre-LHC fits, diamonds for fits including the first SUSY searches by CMS and ATLAS, triangles including all relevant 2010 LHC data, squares for estimates of the impacts of LHC data sets with $1,2,7 \mathrm{fb}^{-1}$ in the absence of a SUSY discovery, and open stars for fits including $1 \mathrm{fb}^{-1}$ of LHC data. Also shown as crosses are two older benchmark points: SPS1a [89] and a similar benchmark point $\mathrm{B}^{\prime \prime}[113,114]$. The various symbols are also coded with different colours for different fitting groups.

It is also reassuring that the pre-LHC stars lie close together, as do the post-LHC fits. As expected, we see that the best-fit value of $m_{1 / 2}$ increases monotonically with the continuing experimental absence of SUSY. We note that the best-fit values of $m_{0}$ and $\tan \beta$ also tend to increase, ${ }^{5}$ which is due to the interplay of the LHC constraints with $(g-2)_{\mu}$, in particular. Reconciling the larger $m_{1 / 2}$ enforced by the LHC limits with $(g-2)_{\mu}$ tends to require larger $\tan \beta$, and

\footnotetext{
${ }^{5}$ We note in passing that, even before the LHC data, the value $\tan \beta=3$ often used as a default in experimental analyses was already disfavoured by all global fits.
}

avoiding a charged LSP then tends to require larger $m_{0}$, as seen in Fig. 2. On the other hand, $A_{0}{ }^{6}$ exhibits a weak tendency to decrease, though it is not tightly constrained by current data. $^{7}$

The fit probability, as evaluated from the $\chi^{2} /$ dof, decreases monotonically, though it has not yet fallen sufficiently far to call the CMSSM into question. The decreasing probability is also due to the increasing tension between $(g-2)_{\mu}$ and other low-energy data on the one side and the non-observation of SUSY particles on the other side. The curved trend lines are the projections in the $\left(m_{0}, m_{1 / 2}\right)$ and $\left(m_{1 / 2}, \tan \beta\right)$ planes of a least-squares fit to the best-fit points.

We propose below updated benchmark planes, lines and points for the CMSSM. However, the fact that this model has come under some pressure from the limits of the LHC SUSY searches motivates putting a stronger emphasis than in the past on alternative SUSY models, some of which are discussed subsequently.

\subsection{Benchmark planes, lines and points in the CMSSM}

Motivated by the $(g-2)_{\mu}$ and $b \rightarrow s \gamma$ constraints, here we fix $\mu>0$ and propose to consider $\left(m_{0}, m_{1 / 2}\right)$ planes for $\tan \beta=10$ and 40 , which bracket the range of $\tan \beta$ favoured

\footnotetext{
${ }^{6}$ The definition of $A_{0}$, as explained previously in the text, has to be kept in mind! Many public codes use the opposite sign convention for $A_{0}$.

${ }^{7}$ For this reason, and because $A_{0}$ is of secondary importance for most of the spectrum, we do not discuss its behaviour in detail.
} 
Fig. 1 (Color online) CMSSM fit points are projected on (upper panel) the $\left(m_{0}, m_{1 / 2}\right)$ plane and (lower panel) the $\left(m_{1 / 2}, \tan \beta\right)$ plane. The best-fit points for different data sets are indicated by different symbols: closed stars for pre-LHC fits (Allanach [96], Fittino [97], MC [98], SuperBayes [99]), diamonds for fits including the first SUSY searches by CMS and ATLAS (Allanach [96], MC [98]), triangles including all relevant 2010 LHC data (Allanach [100], Bertone [101], Fittino [102], MC [103]), squares for estimates of the impacts of LHC data sets with $1,2,7 \mathrm{fb}^{-1}$ in the absence of a SUSY discovery [97], and open stars for fits including $1 \mathrm{fb}^{-1}$ of LHC data [110-112]. Also shown as crosses are two older benchmark points: SPS1a [89] and a similar benchmark point $B^{\prime \prime}[113,114]$. The various symbols are also coded with different colours for different fitting groups, as shown in the legend. The line illustrates the trend of these fits as stronger constraints are incorporated
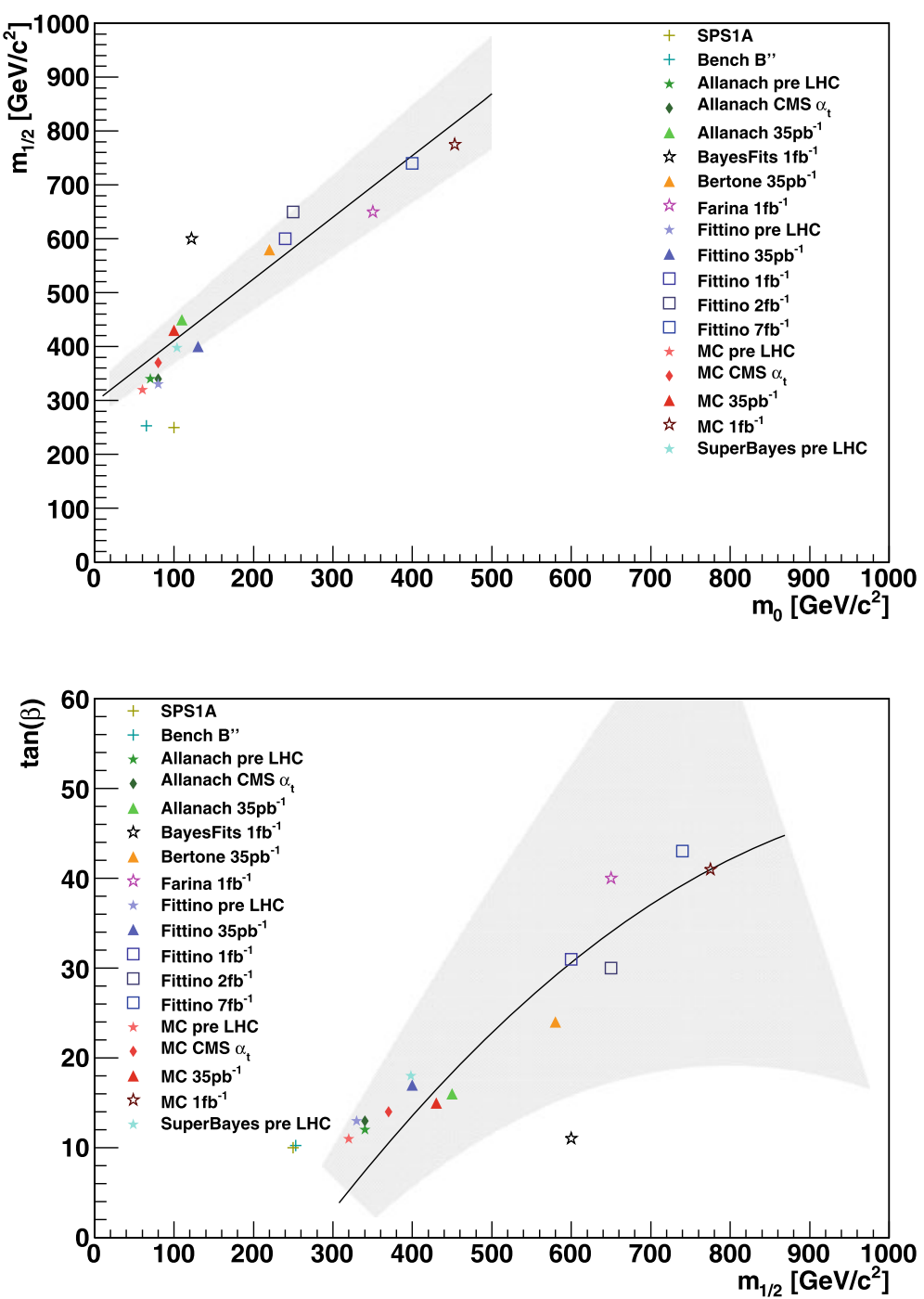

by the trend of global fits to data before and after the start of the LHC, as shown in Fig. 2.

\subsubsection{CMSSM plane I}

$\left(m_{0}, m_{1 / 2}\right)$ plane:

$\mu>0, \quad \tan \beta=10, \quad A_{0}=0$.

Since present fits provide only weak indications on the possible range of $A_{0}$, while favouring slightly values that become increasingly negative as $m_{1 / 2}, m_{0}$ and $\tan \beta$ increase, we propose to consider $A_{0}=0$ for the $\left(m_{0}, m_{1 / 2}\right)$ plane with $\tan \beta=10$.

\subsubsection{CMSSM plane II}

$\underline{\left(m_{0}, m_{1 / 2}\right) \text { plane: }}$

$\mu>0, \quad \tan \beta=40, \quad A_{0}=-500 \mathrm{GeV}$.
The value $A_{0}=-500 \mathrm{GeV}$ is proposed for the $\left(m_{0}, m_{1 / 2}\right)$ plane with $\tan \beta=40$, so as to mirror the trend towards negative $A_{0}$ seen in global fits.

\subsubsection{CMSSM lines and points}

As well as these planes, as also seen in Fig. 2 we propose lines and points lying on them, that might be useful for future dedicated detector studies and comparisons. The co-ordinates of some of the proposed points lie close to the trend line shown in Fig. 1, but the lines also include points in different regions of the $\left(m_{0}, m_{1 / 2}\right)$ planes as shown in the Tables below. The lines are chosen to illustrate a range of phenomenological possibilities for sparticle mass hierarchies and cascade decay branching ratios, providing a greater variety of models and SUSY phenomenology than is covered by the CMSSM fits alone. The points are evenly spaced along the lines, and some of the lower-mass points are already excluded by the early LHC direct SUSY 


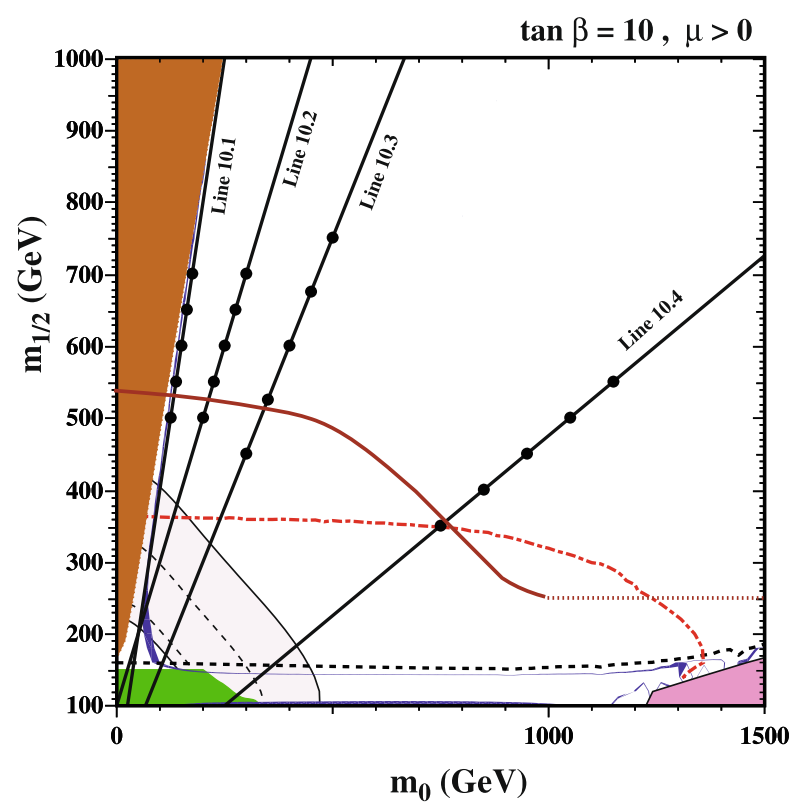

Fig. 2 (Color online) The CMSSM $\left(m_{0}, m_{1 / 2}\right)$ planes for (left) $\tan \beta=10, \mu>0$ and $A_{0}=0$, and (right) $\tan \beta=40, \mu>0$ and $A_{0}=-500 \mathrm{GeV}$. In the brown shaded regions at small $m_{0}$ the LSP is charged, in the pink shaded regions at large $m_{0}$ there is no consistent electroweak vacuum, the green shaded regions are excluded by $b \rightarrow s \gamma$, and the grey shaded regions are favoured by $(g-2)_{\mu}$ at the 1- (2-) $\sigma$ level indicated by dashed (solid) lines. LEP searches for charginos exclude the regions below the near-horizontal black dashed

searches, whilst others are not. At any one time, one can define the 'active point' along any line to be the lightest one which is not ruled out by LHC direct SUSY searches at the 95\% confidence level. In this way, if the SUSY exclusion limits advance, the points we define should remain useful, in particular because experimental signatures are similar for different points along the same line.

In the case of plane I with $\tan \beta=10$, we propose to consider four embedded lines, which yield different possibilities for the most important sparticle cascade decay branching ratios. Some properties of the reference points, obtained from the Suspect [69]/Sdecay [115] computer codes, are shown in Tables 2-5. The parameter range for the points on each line was chosen to yield production cross sections, summed over the various squark and gluino channels, in the range of few-100 fb, consistent with the reach of the ongoing LHC run. The main qualitative features of these lines are discussed here.

Line 10.1 is defined by $m_{0}=0.25 \times m_{1 / 2}$, with reference points spaced in steps of $\Delta m_{1 / 2}=50 \mathrm{GeV}$. Along this line the gluino is heavier than the squarks, yielding a priori relatively higher fractions of final states with fewer hadronic jets, and the branching ratios for $\tilde{\chi}_{2}^{0}$ decays into $\ell \equiv e / \mu$ and $\tau$ (s)lepton flavours are relatively high, offering interesting prospects for dilepton signatures.

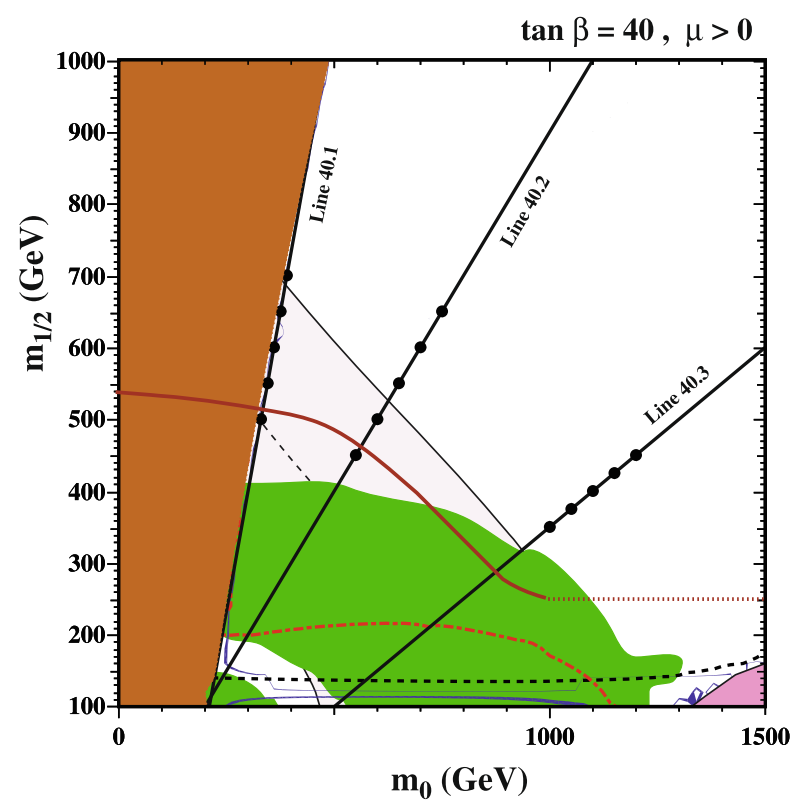

lines, LEP searches for the Higgs boson exclude the regions below the near-horizontal red dot-dashed lines, and LHC searches exclude the regions below the purple lines. The benchmark lines are solid black, and the dots denote the benchmark points spaced regularly along these lines. The dark blue strips yield the correct cold dark matter density in the CMSSM. All experimental numbers and the corresponding references can be found in [103]

Line 10.2 is defined by $\boldsymbol{m}_{\mathbf{0}}=\mathbf{0 . 5} \times \boldsymbol{m}_{\mathbf{1} / \mathbf{2}}-\mathbf{5 0} \mathrm{GeV}$, with reference points spaced in steps of $\Delta m_{1 / 2}=50 \mathrm{GeV}$. Along this line, the gluino is again heavier than the squarks, yielding a priori relatively higher fractions of final states with fewer hadronic jets. However, the branching ratio for $\tilde{\chi}_{2}^{0}$ decays into the $e / \mu$ flavours is much smaller than that for the $\tau$ (s)lepton flavour.

Line 10.3 is defined by $m_{1 / 2}=1.5 \times m_{0}$, with reference points spaced in steps of $\Delta m_{0}=50 \mathrm{GeV}$. Along this line, once more the gluino is heavier than the squarks. However, the pattern of $\tilde{\chi}_{2}^{0}$ decays is completely different, with $\tilde{\chi}_{2}^{0} \rightarrow \tilde{\chi}_{1}^{0} h$ decays becoming dominant, offering an interesting possible discovery channel for the lightest MSSM Higgs boson.

Line 10.4 is defined by $m_{0}=2 \times m_{1 / 2}+50 \mathrm{GeV}$, with reference points spaced in steps of $\Delta m_{1 / 2}=50 \mathrm{GeV}$. Along this line, the gluino is lighter than the spartners of the lighter quark flavours, yielding a priori relatively higher fractions of final states with more hadronic jets. The lighter stop and sbottom squarks are significantly lighter than the other squarks, with the $\tilde{t}_{1}$ even lighter than the gluino though not always light enough to overcome the kinematic restriction on direct $\tilde{g} \rightarrow \tilde{t}_{1} t$ decays. However, the branching ratios for three-body $\tilde{g} \rightarrow \tilde{\chi}_{1}^{0} \bar{t} t, \tilde{\chi}_{1}^{ \pm} \bar{t} b$ decays are dominant along this line. 
Table 2 Line 10.1

$\tan \beta=10, A_{0}=0, m_{0}=$

$0.25 \times m_{1 / 2}, \Delta m_{1 / 2}=50 \mathrm{GeV}$ (masses in $\mathrm{GeV}$, rounded to $5 \mathrm{GeV}$ accuracy; branching ratios in \%)

\begin{tabular}{lllllll}
\hline Point & $m_{1 / 2}$ & $m_{0}$ & $m_{\tilde{g}}$ & $\left\langle m_{\tilde{q}}\right\rangle$ & $\mathrm{BR}\left(\tilde{\chi}_{2}^{0} \rightarrow \tilde{\ell} \ell\right)$ & $\mathrm{BR}\left(\tilde{\chi}_{2}^{0} \rightarrow \tilde{\tau} \tau\right)$ \\
\hline 10.1 .1 & 500 & 125 & 1145 & 1030 & 24 & 19 \\
10.1 .2 & 550 & 137.5 & 1255 & 1125 & 26 & 18 \\
10.1 .3 & 600 & 150 & 1355 & 1220 & 28 & 18 \\
10.1 .4 & 650 & 162.5 & 1460 & 1310 & 28 & 17 \\
10.1 .5 & 700 & 175 & 1565 & 1405 & 29 & 17 \\
$10.1 . \mathrm{N}$ & $\ldots$ & $\ldots$ & $\ldots$ & $\ldots$ & $\ldots$ & $\ldots$ \\
\hline
\end{tabular}

Table 3 Line 10.2:

$\tan \beta=10, A_{0}=0, m_{0}=$ $0.5 \times m_{1 / 2}-50 \mathrm{GeV}, \Delta m_{1 / 2}=$ $50 \mathrm{GeV}$ (masses in $\mathrm{GeV}$, rounded to $5 \mathrm{GeV}$ accuracy; branching ratios in \%)
Table 4 Line 10.3:

$\tan \beta=1 \overline{0, A_{0}=0,} m_{1 / 2}=$ $1.5 \times m_{0}, \Delta m_{0}=50 \mathrm{GeV}$ (masses in $\mathrm{GeV}$, rounded to $5 \mathrm{GeV}$ accuracy; branching ratios in \%)

\begin{tabular}{lllllll}
\hline Point & $m_{1 / 2}$ & $m_{0}$ & $m_{\tilde{g}}$ & $\left\langle m_{\tilde{q}}\right\rangle$ & $\mathrm{BR}\left(\tilde{\chi}_{2}^{0} \rightarrow \tilde{\ell} \ell\right)$ & $\mathrm{BR}\left(\tilde{\chi}_{2}^{0} \rightarrow \tilde{\tau} \tau\right)$ \\
\hline 10.2 .1 & 500 & 200 & 1150 & 1045 & 3 & 40 \\
10.2 .2 & 550 & 225 & 1255 & 1140 & 3 & 37 \\
10.2 .3 & 600 & 250 & 1360 & 1235 & 2 & 34 \\
10.2 .4 & 650 & 275 & 1465 & 1330 & 3 & 32 \\
10.2 .5 & 700 & 300 & 1570 & 1425 & 3 & 30 \\
$10.2 . \mathrm{N}$ & $\ldots$ & $\ldots$ & $\ldots$ & $\ldots$ & $\ldots$ & $\ldots$ \\
\hline
\end{tabular}

\begin{tabular}{llllll}
\hline Point & $m_{1 / 2}$ & $m_{0}$ & $m_{\tilde{g}}$ & $\left\langle m_{\tilde{q}}\right\rangle$ & $\mathrm{BR}\left(\tilde{\chi}_{2}^{0} \rightarrow \tilde{\chi}_{1}^{0} h\right)$ \\
\hline 10.3 .1 & 450 & 300 & 1050 & 975 & 92 \\
10.3 .2 & 525 & 350 & 1210 & 1125 & 92 \\
10.3 .3 & 600 & 400 & 1370 & 1275 & 92 \\
10.3 .4 & 675 & 450 & 1525 & 1420 & 92 \\
10.3 .5 & 750 & 500 & 1680 & 1565 & 92 \\
$10.3 . \mathrm{N}$ & $\ldots$ & $\ldots$ & $\ldots$ & $\ldots$ & $\ldots$ \\
\hline
\end{tabular}

Table 5 Line 10.4:

$\tan \beta=10, A_{0}=0, m_{0}=$ $2 \times m_{1 / 2}+50 \mathrm{GeV}$, $\Delta m_{1 / 2}=50 \mathrm{GeV}$ (masses in $\mathrm{GeV}$, rounded to $5 \mathrm{GeV}$ accuracy; branching ratios in \%)

\begin{tabular}{llllllll}
\hline Point & $m_{1 / 2}$ & \multicolumn{1}{c}{$m_{0}$} & \multicolumn{1}{c}{$m_{\tilde{g}}$} & $\left\langle m_{\tilde{q}}\right\rangle$ & \multicolumn{1}{c}{$m_{\tilde{t}_{1}}$} & $m_{\tilde{b}_{1}}$ & $\mathrm{BR}\left(\tilde{g} \rightarrow \tilde{\chi}^{0} \bar{t} t, \tilde{\chi}^{ \pm} \bar{t} b\right)$ \\
\hline 10.4 .1 & 350 & 750 & 870 & 1040 & 720 & 915 & 53 \\
10.4 .2 & 400 & 850 & 985 & 1175 & 815 & 1035 & 71 \\
10.4 .3 & 450 & 950 & 1095 & 1310 & 910 & 1155 & 100 \\
10.4 .4 & 500 & 1050 & 1205 & 1445 & 1010 & 1275 & 100 \\
10.4 .5 & 550 & 1150 & 1320 & 1580 & 1105 & 1395 & 100 \\
$10.4 . \mathrm{N}$ & $\ldots$ & $\ldots$ & $\ldots$ & $\ldots$ & $\ldots$ & $\ldots$ & $\ldots$ \\
\hline
\end{tabular}

For the plane II with $\tan \beta=40$ and $A_{0}=-500 \mathrm{GeV}$, we propose three embedded lines. Some properties of the reference points are shown in Tables $6-8$, and their main qualitative features are discussed here.

Line 40.1 is defined by $m_{0}=0.3 \times m_{1 / 2}+180 \mathrm{GeV}$, with reference points spaced in steps of $\Delta m_{1 / 2}=50 \mathrm{GeV}$. Some properties of the reference points are shown in Table 6 . Along this line, the gluino and squarks have very similar masses, and the gluino has large branching ratios for decays into $\tilde{\chi}_{1}^{0} \bar{t} t$ and $\tilde{\chi}_{1}^{ \pm} \bar{t} b$ final states. The $\tilde{\tau}_{1} \tau$ final state dominates $\tilde{\chi}_{2}^{0}$ decays, with $\tilde{\ell} \ell$ for $\ell=e, \mu$ being suppressed because of the larger masses of the $\tilde{\ell}$ for this value of $\tan \beta$.
Line 40.2 is defined by $m_{0}=m_{1 / 2}+100 \mathrm{GeV}$, with reference points spaced in steps of $\Delta m_{1 / 2}=50 \mathrm{GeV}$. Some properties of the reference points are shown in Table 7, where we indicate separately the minimum and maximum mass values of the spartners of the light quark flavours, which bracket the gluino mass and are very close to it. We also show the masses of the $\tilde{t}_{1}$ and $\tilde{b}_{1}$. Since these are significantly lighter, the two-body gluino decays to $\tilde{t}_{1} \bar{t}$ and $\tilde{b}_{1} \bar{b}$ dominate, leading to $\tilde{\chi}^{0} \bar{t} t$ and $\tilde{\chi}^{ \pm} t b$ final states, as seen previously along Line 10.3. Line 40.2 also features a very large branching ratio for $\tilde{\chi}_{2}^{0} \rightarrow \tilde{\chi}_{1}^{0} h$ decay. 
Table $6 \underline{\text { Line 40.1: }} \tan \beta=40, A_{0}=-500 \mathrm{GeV}, m_{0}=0.3 \times m_{1 / 2}+180 \mathrm{GeV}, \Delta m_{1 / 2}=50 \mathrm{GeV}$ (masses in GeV, rounded to $5 \mathrm{GeV}$ accuracy; branching ratios in \%)

\begin{tabular}{llllllrlll}
\hline Point & $m_{1 / 2}$ & $m_{0}$ & $m_{\tilde{g}}$ & $\left\langle m_{\tilde{q}}\right\rangle$ & $m_{\tilde{t}_{1}}$ & $m_{\tilde{b}_{1}}$ & $\mathrm{BR}\left(\tilde{\chi}_{2}^{0} \rightarrow \tilde{\tau}_{1} \tau / h X\right)$ & $\operatorname{BR}(\tilde{g} \rightarrow \tilde{t} t)$ & $\mathrm{BR}(\tilde{g} \rightarrow \tilde{b} b)$ \\
\hline 40.1 .1 & 500 & 330 & 1155 & 1075 & 780 & 920 & $96 / 3$ & 31 & 40 \\
40.1 .2 & 550 & 345 & 1260 & 1170 & 860 & 1005 & $96 / 4$ & 35 & 39 \\
40.1 .3 & 600 & 360 & 1360 & 1260 & 935 & 1090 & $96 / 4$ & 40 & 33 \\
40.1 .4 & 650 & 375 & 1470 & 1355 & 1015 & 1175 & $94 / 5$ & 40 & 32 \\
40.1 .5 & 700 & 390 & 1570 & 1445 & 1090 & 1260 & $95 / 4$ & $\ldots$ & $\ldots$ \\
$40.1 . \mathrm{N}$ & $\ldots$ & $\ldots$ & $\ldots$ & $\ldots$ & $\ldots$ & $\ldots$ & $\ldots$ & $\ldots$ & $\ldots$ \\
\hline
\end{tabular}

Table 7 Line 40.2: $\tan \beta=40, A_{0}=-500 \mathrm{GeV}, m_{0}=m_{1 / 2}+100 \mathrm{GeV}, \Delta m_{1 / 2}=50 \mathrm{GeV}$ (masses in GeV, rounded to $5 \mathrm{GeV}$ accuracy; branching ratios in \%)

\begin{tabular}{|c|c|c|c|c|c|c|c|c|c|}
\hline Point & $m_{1 / 2}$ & $m_{0}$ & $m_{\tilde{g}}$ & $m_{\tilde{q}}(\min / \max )$ & $m_{\tilde{t}_{1}}$ & $m_{\tilde{b}_{1}}$ & $\operatorname{BR}\left(\tilde{\chi}_{2}^{0} \rightarrow \tilde{\chi}_{1}^{0} h\right)$ & $\mathrm{BR}(\tilde{g} \rightarrow \tilde{t} t)$ & $\mathrm{BR}(\tilde{g} \rightarrow \tilde{b} b)$ \\
\hline 40.2 .1 & 450 & 550 & 1065 & $1060 / 1095$ & 750 & 900 & 87 & 52 & 48 \\
\hline 40.2 .2 & 500 & 600 & 1170 & $1165 / 1200$ & 835 & 995 & 88 & 54 & 46 \\
\hline 40.2 .3 & 550 & 650 & 1280 & $1270 / 1305$ & 915 & 1090 & 89 & 56 & 44 \\
\hline 40.2 .4 & 600 & 700 & 1385 & $1370 / 1415$ & 1000 & 1185 & 90 & 57 & 42 \\
\hline 40.2 .5 & 650 & 750 & 1495 & $1470 / 1520$ & 1085 & 1280 & 91 & 58 & 41 \\
\hline 40.2.N & $\ldots$ & $\ldots$ & $\ldots$ & $\ldots$ & $\ldots$ & $\ldots$ & $\ldots$ & $\ldots$ & $\ldots$ \\
\hline
\end{tabular}

Table $8 \underline{\text { Line 40.3}}: \tan \beta=40, A_{0}=-500 \mathrm{GeV}, m_{0}=2 \times m_{1 / 2}+300 \mathrm{GeV}, \Delta m_{0}=50 \mathrm{GeV}$ (masses in GeV, rounded to $5 \mathrm{GeV}$ accuracy; branching ratios in \%)

\begin{tabular}{lllllllll}
\hline Point & $m_{1 / 2}$ & $m_{0}$ & $m_{\tilde{g}}$ & $\left\langle m_{\tilde{q}}\right\rangle$ & $m_{\tilde{t}_{1}}$ & $m_{\tilde{b}_{1}}$ & $\mathrm{BR}\left(\tilde{\chi}_{2}^{0} \rightarrow \tilde{\chi}_{1}^{0} h^{0}\right)$ & $\mathrm{BR}\left(\tilde{g} \rightarrow \tilde{\chi}^{0} \bar{t} t, \tilde{\chi}^{ \pm} \bar{t} b\right)$ \\
\hline 40.3 .1 & 350 & 1000 & 890 & 1225 & 775 & 960 & 81 & 47 \\
40.3 .2 & 375 & 1050 & 950 & 1295 & 825 & 1020 & 84 & 51 \\
40.3 .3 & 400 & 1100 & 1005 & 1365 & 875 & 1075 & 85 & 56 \\
40.3 .4 & 425 & 1150 & 1065 & 1435 & 925 & 1135 & 86 & 61 \\
40.3 .5 & 450 & 1200 & 1125 & 1505 & 975 & 1195 & 87 & $\ldots 6$ \\
$40.3 . \mathrm{N}$ & $\ldots$ & $\ldots$ & $\ldots$ & $\ldots$ & $\ldots$ & $\ldots$ & $\ldots$ & $\ldots$ \\
\hline
\end{tabular}

Line 40.3 is defined by $m_{0}=2 \times m_{1 / 2}+300 \mathrm{GeV}$, with reference points spaced in steps of $\Delta m_{1 / 2}=50 \mathrm{GeV}$. Some properties of the reference points are shown in Table 8 . Along this line, the branching ratios for gluino decays into light-flavour squarks and stop/sbottom 3-body decays are comparable, as was the case along Line 10.4. Like Line 40.2, Line 40.3 has a large branching ratio for $\tilde{\chi}_{2}^{0} \rightarrow \tilde{\chi}_{1}^{0} h^{0}$ decays.

\subsection{Benchmark planes, lines and points in the NUHM1 and NUHM2}

In defining the above benchmarks in the CMSSM, we have not taken into account the relic dark matter density constraint. As is well known, if one assumes standard Big-Bang cosmology, the accuracy with which the dark matter density is determined essentially reduces the dimensionality of the CMSSM parameter space by one. ${ }^{8}$ For this reason, any $\left(m_{0}, m_{1 / 2}\right)$ plane such as those defined above contains only very narrow strips compatible with this constraint, shown in dark blue in Fig. 2, flanked by regions where the supersymmetric relic is either overdense or underdense. As a corollary, a generic line in a CMSSM plane will yield the appropriate relic density only at a (discrete set of) point(s). The density constraints could be relaxed by postulating, e.g., another contribution to the dark matter in the apparently underdense regions of parameter space, or a deviation from standard Big-Bang cosmology to reduce the relic density in the

${ }^{8}$ This is true if one fixes other, non-CMSSM, but otherwise relevant parameters, in particular the mass of the top quark $m_{t}$ or $\alpha_{s}$. Allowing them to vary (even within their respective $1 \sigma$ uncertainties) has a noticeable effect on the relic abundance, especially, although not only, in the regime of large $m_{0}$. 
apparently overdense regions, or possibly by invoking some form of $R_{p}$ violation that renders the LSP unstable.

The NUHM1 and NUHM2 offer one or two additional parameters that, in general, enter into the calculation of the relic density. Hence, they offer in principle an alternative possibility for adjusting the relic density to respect the cosmological constraint in extended regions of a benchmark $\left(m_{0}, m_{1 / 2}\right)$ plane, or along segments of the embedded benchmark lines, by adjusting one or both of the degrees of non-universality of the SSB Higgs mass parameters. Varying these parameters does not impact greatly the sensitivities of the primary LHC missing-energy searches for SUSY, though it may affect the sensitivity to flavour observables such as $\mathrm{BR}(b \rightarrow s \gamma)$ and $\mathrm{BR}\left(B_{s} \rightarrow \mu^{+} \mu^{-}\right)$, and certainly impacts the predictions for and constraints imposed by Higgs searches [98, 103].

The study of these signatures lies beyond the scope of this document. For our purposes here, it is sufficient to note that the CMSSM models discussed above could also be taken as benchmarks for a more detailed study of their extensions to the NUHM1 and/or NUHM2 with $M_{A}$ and/or $\mu$ adjusted to fulfill the CDM constraint. With this idea in mind, we have explored what value of $\mu$ would bring the relic density into the WMAP range for each of the CMSSM lines and points defined above.

In the case of Line 10.1, this exercise is unnecessary, since the coannihilation strip for $\tan \beta=10$ and $A_{0}=0$ has the slope $m_{0}=0.25 \times m_{1 / 2}$. In the case of Line 10.2, a suitable choice is: ${ }^{9}$

NUHM Line 10.2: $\quad \mu=80 \mathrm{GeV}+0.42 \times m_{1 / 2}$,

for Line $\mathbf{1 0 . 3}$ one may choose

NUHM Line 10.3: $\quad \mu=74 \mathrm{GeV}+0.43 \times m_{1 / 2}$,

and for Line 10.4 one may choose

NUHM Line 10.4: $\quad \mu=68 \mathrm{GeV}+0.44 \times m_{1 / 2}$,

though the linear approximation for $\mu$ is less accurate in this case.

The NUHM1 modification of Line 10.2 produces some changes in the branching ratios listed in Table 3. The $\tilde{\chi}_{2}^{0}$ decays are dominated by the $\tilde{\tau} \tau$ final state only in the case of 10.2.1 and 10.2.2. For 10.2.3 and 10.2.4 $\tilde{\chi}_{2}^{0}$ mostly undergoes 3-body $\tilde{\chi}_{1}^{0} f \bar{f}$ decays mediated by an off-shell $Z^{*} / \gamma$. For 10.2.5 and beyond, $\tilde{\chi}_{2}^{0} \rightarrow \tilde{\chi}_{1}^{0} Z$. In all of these cases, gluinos mostly decay to $\tilde{t} t$ and $\tilde{b} b$ pairs, as for the CMSSM

\footnotetext{
${ }^{9}$ This linear fit, and the following ones, are approximate, with a small dependence of the numerical values of the coefficients on the code used for the renormalization-group evolution.
}

10.2.X points. The modification of Line 10.3 has the consequence that the decay mode $\tilde{\chi}_{2}^{0} \rightarrow \tilde{\chi}_{1}^{0} h$ is no longer kinematically accessible: $\tilde{\chi}_{2}^{0} \rightarrow \tilde{\chi}_{1}^{0} f \bar{f}$ via an off-shell $Z^{*} / \gamma$ for points $1-4$, while $\tilde{\chi}_{2}^{0} \rightarrow \tilde{\chi}_{1}^{0} Z$ for 10.3 .5 and beyond. In the case of Line 10.4, $\operatorname{BR}(\tilde{g} \rightarrow \tilde{t} t)=1$.

The coannihilation strip for $\tan \beta=40$ and $A_{0}=$ $-500 \mathrm{GeV}$ may be parametrized by $m_{0}=171 \mathrm{GeV}+$ $0.318 m_{1 / 2}$. This is very close to our CMSSM Line 40.1, whose reference points provide acceptable fits to the relic density. In the case of Line 40.2, the dark matter density is approximately satisfied for

NUHM Line 40.2: $\quad \mu=95 \mathrm{GeV}+0.4 \times m_{1 / 2}$,

and for Line $\mathbf{4 0 . 3}$ by

NUHM Line 40.3: $\quad \mu=50 \mathrm{GeV}+0.48 \times m_{1 / 2}$,

though with nonlinear deviations at higher $m_{1 / 2}$.

As in the case of the $\tan \beta=10$ reference points, the change of $\mu$ values for Lines 40.2 and 40.3 has the consequence that the decay mode $\tilde{\chi}_{2}^{0} \rightarrow \tilde{\chi}_{1}^{0} h$ is no longer kinematically accessible, and $\tilde{\chi}_{2}^{0} \rightarrow \tilde{\chi}_{1}^{0} f \bar{f}$, via an off-shell $Z^{*} / \gamma$, is the dominant decay mode. The gluino decays remain dominated by stop and sbottom final states, with twobody $\tilde{g} \rightarrow \tilde{Q} Q(Q=t, b)$ modes for Line 40.2 , and mostly 3-body $\tilde{g} \rightarrow \tilde{\chi}^{0( \pm)} \tilde{Q} Q^{(\prime)}$ modes for Line 40.3 .

\subsection{Planes, lines and points in mGMSB}

We propose two $\left(M_{\text {mess }}, \Lambda\right)$ planes, distinguished by the identity of the NLSP, giving different final state topologies.

\subsection{1 mGMSB plane I}

$\left(\Lambda, M_{\text {mess }}\right)$ plane:

$c_{\text {grav }}=1, \quad N_{\text {mess }}=3, \quad \tan \beta=15, \quad \mu>0$.

This plane corresponds to a stau NLSP, thus signatures include di-taus plus missing energy plus jets.

\subsection{2 mGMSB plane II}

$\left(\Lambda, M_{\text {mess }}\right)$ plane: This $\left(M_{\text {mess }}, \Lambda\right)$ plane is defined by:

$c_{\text {grav }}=1, \quad N_{\text {mess }}=1, \quad \tan \beta=15, \quad \mu>0$.

This plane corresponds to a neutralino NLSP, which may decay into a photon and a gravitino inside the detector if the decay is prompt enough. Thus, signatures include jets, missing energy and di-photons. 


\subsection{3 mGMSB lines and points}

Here, we employ SOFTSUSY to produce the spectra, which are successively heavier for each point. The decay branching ratios are calculated by Sdecay [115] for line mGMSB2.1 and HERWIG++-2.5.1 [116] for line mGMSB1 and mGMSB2.2.

Line mGMSB1 is defined from mGMSB plane I by $N_{\text {mess }}=3, \tan \beta=15, \mu>0, \Lambda=M_{\text {mess }} / 2$ with $\Delta M_{\text {mess }}=$ $10 \mathrm{TeV}$. Table 9 shows some salient features of the spectrum and decays. The point mGMSB 1.2 corresponds to the SPS 7 benchmark point [89].

It is clear from Table 9 that many signal events should have di-lepton end points and missing transverse momentum from cascade decays involving the lightest neutralinos, as well as other events involving taus coming from the prompt stau NLSP decays into taus and gravitinos.

Line mGMSB2.1 is defined from mGMSB plane II by $N_{\text {mess }}=1, \tan \beta=15, \mu>0, \Lambda=0.9 M_{\text {mess }}$ with $\Delta M_{\text {mess }}=10 \mathrm{TeV}$. Table 10 shows some salient features of the spectrum and decays. The squark masses are larger than the gluino mass, meaning that the multiplicity of jets will be higher, on average. Also, the possibility of producing $Z \mathrm{~s}$ from the prompt neutralino NLSP decays for the heavier points allows an additional handle on the events: one could search for jets, $\gamma, Z$ and missing transverse momentum.

Line mGMSB2.2 is defined from mGMSB plane II by $N_{\text {mess }}=1, \tan \beta=15, \mu>0, M_{\text {mess }}=10^{9} \mathrm{GeV}$ and $\Delta \Lambda=10 \mathrm{TeV}$. This line has a quasi-stable neutralino NLSP, with average decay lengths of kilometers, resulting in missing transverse momentum signatures. Table 11 shows some salient features of the spectrum and decays. The squark masses are larger than the gluino mass, meaning that the multiplicity of jets will be higher, on average. The lightest $\mathrm{CP}$-even Higgs is produced in sparticle decay chains in association with jets, starting from gluinos, as shown in the last two columns of the table.

\subsection{Planes, lines and points in mAMSB}

We will define one $\left(m_{\text {aux }}, m_{0}\right)$ plane, which contains the SPS 9 benchmark point. Furthermore we include the model line defined in [89].

\subsection{1 mAMSB plane I}

This plane is adopted from SPS 9.

$\underline{\left(m_{\text {aux }}, m_{0}\right) \text { plane: }}$

$\tan \beta=10, \quad \mu>0$.

\subsection{2 mAMSB line and points}

The line is defined on mAMSB plane I above.

Line mAMSB1 has $\tan \beta=10, \mu>0, m_{0}=0.0075 m_{\text {aux }}$. The discrete points have $m_{\mathrm{aux}}$ as an integer multiple of $10 \mathrm{TeV}$, starting from $40 \mathrm{TeV}$. Some features of the spectrum of the first few points (obtained with SOFTSUSY) are
Table 9 Line mGMSB1:

$N_{\text {mess }}=3, \tan \beta=15, \mu>0$, $\Delta M_{\text {mess }}=10 \mathrm{TeV}$ (masses in $\mathrm{GeV}$, rounded to $5 \mathrm{GeV}$ accuracy, branching ratios in $\%) . l$ stands for charged leptons of the first two generations

Table 10 Line mGMSB2.1: $N_{\text {mess }}=1, \tan \beta=15, \mu>0$, $\Delta M_{\text {mess }}=10 \mathrm{TeV}$ (masses in $\mathrm{GeV}$, rounded to $5 \mathrm{GeV}$ accuracy, branching ratios in \%)

\begin{tabular}{lccccccc}
\hline Point & $M_{\text {mess }} / \mathrm{TeV}$ & $m_{\tilde{\tau}_{1}}$ & $m_{\chi_{1}^{0}}$ & $m_{\tilde{g}}$ & $\left\langle m_{\tilde{q}}\right\rangle$ & $\mathrm{BR}\left(\chi_{1}^{0} \rightarrow \tilde{l}_{R} l\right)$ & $\mathrm{BR}\left(\tilde{l}_{R} \rightarrow l \tilde{G}\right)$ \\
\hline mGMSB1.1 & 70 & 110 & 140 & 840 & 785 & 56 & 3 \\
mGMSB1.2 & 80 & 125 & 165 & 950 & 885 & 59 & 8 \\
mGMSB1.3 & 90 & 140 & 185 & 1055 & 985 & 61 & 17 \\
mGMSB1.4 & 100 & 155 & 205 & 1160 & 1080 & 62 & 32 \\
mGMSB1.5 & 110 & 170 & 230 & 1265 & 1180 & 62 & 47 \\
mGMSB1.6 & 120 & 185 & 250 & 1370 & 1285 & 63 & $\ldots 1$ \\
mGMSB1.N & $\ldots$ & $\ldots$ & $\ldots$ & $\ldots$ & $\ldots$ & $\ldots$ & $\ldots$
\end{tabular}

\begin{tabular}{|c|c|c|c|c|c|c|}
\hline Point & $M_{\mathrm{mess}} / \mathrm{TeV}$ & $m_{\chi_{1}^{0}}$ & $m_{\tilde{g}}$ & $\left\langle m_{\tilde{q}}\right\rangle$ & $\mathrm{BR}\left(\chi_{1}^{0} \rightarrow \tilde{G} \gamma\right)$ & $\operatorname{BR}\left(\chi_{1}^{0} \rightarrow Z^{0} \tilde{G}\right)$ \\
\hline mGMSB2.1.1 & 80 & 115 & 705 & 820 & 99 & 1 \\
\hline mGMSB2.1.2 & 90 & 130 & 785 & 915 & 97 & 3 \\
\hline mGMSB2.1.3 & 100 & 145 & 865 & 1010 & 95 & 5 \\
\hline mGMSB2.1.4 & 110 & 160 & 940 & 1100 & 93 & 7 \\
\hline mGMSB2.1.5 & 120 & 175 & 1020 & 1190 & 90 & 10 \\
\hline mGMSB2.1.6 & 130 & 190 & 1095 & 1280 & 89 & 11 \\
\hline mGMSB2.1.N & $\ldots$ & $\ldots$ & $\ldots$ & $\cdots$ & $\cdots$ & . \\
\hline
\end{tabular}


Table 11 Line mGMSB2.2

$N_{\text {mess }}=1, \tan \beta=15$,

$M_{\text {mess }}=10^{9} \mathrm{GeV}, \mu>0$,

$\Delta \Lambda=10 \mathrm{TeV}$ (masses in GeV, rounded to $5 \mathrm{GeV}$ accuracy, branching ratios in \%)
Table 12 mAMSB: $\tan \beta=$ $10, \mu>0, m_{0}=0.0075 m_{\text {aux }}$ (masses in $\mathrm{GeV}$ rounded to the nearest $5 \mathrm{GeV}$, branching ratios in $\%)$
Table 13 Line MM-AMSB1: $20 \leq m_{3 / 2} \leq 35$ with $\alpha=5$, $\tan \beta=10, \mu>0, n_{i}=1 / 2$, $l_{a}=1$ (sparticle masses in $\mathrm{GeV}$ rounded to the nearest $5 \mathrm{GeV}$ )

\begin{tabular}{lllllll}
\hline Point & $\Lambda / \mathrm{TeV}$ & $m_{\chi_{1}^{0}}$ & \multicolumn{1}{c}{$m_{\tilde{g}}$} & $\left\langle m_{\tilde{q}}\right\rangle$ & $\operatorname{BR}\left(\tilde{g} \rightarrow \chi_{2}^{0} j j\right)$ & $\mathrm{BR}\left(\chi_{2}^{0} \rightarrow \chi_{1}^{0} h\right)$ \\
\hline mGMSB2.2.1 & 120 & 160 & 935 & 1155 & 17 & 48 \\
mGMSB2.2.2 & 130 & 175 & 1005 & 1245 & 17 & 52 \\
mGMSB2.2.3 & 140 & 190 & 1075 & 1330 & 16 & 53 \\
mGMSB2.2.4 & 150 & 200 & 1145 & 1420 & 16 & 55 \\
mGMSB2.2.5 & 160 & 215 & 1215 & 1510 & 15 & 57 \\
mGMSB2.2.6 & 170 & 230 & 1280 & 1595 & 15 & 58 \\
mGMSB2.2.N & $\ldots$ & $\ldots$ & $\ldots$ & $\ldots$ & $\ldots$ & $\ldots$
\end{tabular}

\begin{tabular}{lllllllll}
\hline Point & $m_{\text {aux }}$ & $m_{0}$ & $m_{\tilde{g}}$ & $\left\langle m_{\tilde{q}}\right\rangle$ & $m_{\tilde{t}_{1}}$ & $m_{\tilde{b}_{1}}$ & $\mathrm{BR}(\tilde{g} \rightarrow \tilde{t} t)$ & $\mathrm{BR}(\tilde{g} \rightarrow \tilde{b} b)$ \\
\hline mAMSB1.1 & $4 \times 10^{4}$ & 300 & 890 & 880 & 630 & 765 & 69 & 29 \\
$\operatorname{mAMSB} 1.2$ & $5 \times 10^{4}$ & 375 & 1085 & 1080 & 780 & 940 & 74 & 25 \\
$\operatorname{mAMSB} 1.3$ & $6 \times 10^{4}$ & 450 & 1280 & 1280 & 925 & 1110 & 76 & 24 \\
mAMSB1.N & $\ldots$ & $\ldots$ & $\ldots$ & $\ldots$ & $\ldots$ & $\ldots$ & $\ldots$ & $\ldots$ \\
\hline
\end{tabular}

\begin{tabular}{llllll}
\hline Point & $\alpha$ & $m_{3 / 2}[\mathrm{TeV}]$ & $m_{\tilde{s}}$ & $\left\langle m_{\tilde{q}}\right\rangle$ & $m_{\tilde{\chi}_{1}^{0}}$ \\
\hline MM-AMSB1.1 & 5 & 20 & 1030 & 915 & 430 \\
MM-AMSB1.2 & 5 & 25 & 1270 & 1125 & 550 \\
MM-AMSB1.3 & 5 & 30 & 1505 & 1330 & 665 \\
MM-AMSB1.4 & 5 & 35 & 1740 & 1535 & 780 \\
MM-AMSB1.N & $\ldots$ & $\ldots$ & $\ldots$ & $\ldots$ & $\ldots$ \\
\hline
\end{tabular}

listed in Table 12. The points have production cross sections for gluino and squark final states in the 6-200 $\mathrm{fb}$ range. The point mAMSB1.3 corresponds to the SPS 9 benchmark point [89].

\subsection{Planes, lines and points in the MM-AMSB}

\subsection{1 mAMSB plane I}

The mixed modulus-anomaly-mediated SUSY breaking scenario is characterized by the parameters specified in (8). The parameter $l_{a}$ can take values 0 or 1 depending on whether the gauge field is localized on a $D 3$ or $D 7$ brane, whereas $n_{i}$ can be 0,1 , or $1 / 2$ for a matter field localized on a $D 7$ brane, a $D 3$ brane, or a brane intersection. In the following we focus on a scenario where $l_{a}=1$ for all gauge fields and where $n_{i}=1 / 2$ for all matter fields. Furthermore we fix $\tan \beta$ and the sign of $\mu$ in accordance with the benchmark scenarios in other SUSY breaking models. We thus consider a benchmark plane in the gravitino mass, $m_{3 / 2}$, and the parameter $\alpha$, which determines the relative weight of anomaly and gravity mediation: $\left(m_{3 / 2}, \alpha\right)$ plane:

$\tan \beta=10, \quad \mu>0, \quad n_{i}=1 / 2, \quad l_{a}=1$.

\subsubsection{MM-AMSB lines and points}

For the first line in the MM-AMSB benchmark plane we fix $\alpha$ and vary the gravitino mass:

Line MM-AMSB1: $\alpha=5$ and $20 \leq m_{3 / 2} \leq 35$.

This choice of $\alpha$ results in a relative size of the weak scale gaugino mass parameters of $M_{1}: M_{2}: M_{3} \simeq 1: 1.2: 2.2$, quite different from the CMSSM with gaugino mass unification at the GUT scale and a corresponding weak scale ratio of $M_{1}: M_{2}: M_{3} \simeq 1: 2: 6$. Thus, MM-AMSB models with $\alpha \approx 5$ typically have a more compressed mass spectrum than the CMSSM. Line 1 within the MM-AMSB is characterized by a SPS1a-like phenomenology with a sequence of two-body decays and features various mass edges. Points near line 1 furthermore provide the correct dark matter abundance. A set of benchmark points with squark and gluino masses in the range between 1 and $1.5 \mathrm{TeV}$ is collected in Table 13. The spectrum has been calculated with ISASUGRA [117]. It is interesting to note that it is possible to distinguish the MM-AMSB models on line 1 from the 
Table 14 Line MM-AMSB2:

$10 \leq \alpha \leq 25$ with $\tan \beta=$

$10, \mu>0, n_{i}=1 / 2, l_{a}=1$

(sparticle masses in $\mathrm{GeV}$

rounded to the nearest $5 \mathrm{GeV}$ ).

$m_{3 / 2}$ is fixed such the resulting

squark and gluino mass

spectrum is around $1.2 \mathrm{TeV}$

\begin{tabular}{lcclccc}
\hline Point & $\alpha$ & $m_{3 / 2}[\mathrm{TeV}]$ & $M_{1}: M_{2}: M_{3}$ & $m_{\tilde{g}}$ & $\left\langle m_{\tilde{q}}\right\rangle$ & $m_{\tilde{\chi}_{1}^{0}}$ \\
\hline MM-AMSB2.1 & 10 & 10 & $1: 1.5: 3.3$ & 1240 & 1130 & 355 \\
MM-AMSB2.2 & 15 & 6.5 & $1: 1.6: 3.8$ & 1280 & 1170 & 315 \\
MM-AMSB2.3 & 20 & 4.5 & $1: 1.6: 4.1$ & 1220 & 1115 & 280 \\
MM-AMSB2.4 & 25 & 3.5 & $1: 1.6: 4.3$ & 1205 & 1105 & 260 \\
MM-AMSB2.N & $\ldots$ & $\ldots$ & $\ldots$ & $\ldots$ & $\ldots$ & $\ldots$ \\
\hline
\end{tabular}

CMSSM by using information from mass edges and cross sections [118].

Line MM-AMSB2 features an increasing parameter $\alpha$, resulting in a different ratio of weak scale mass parameters and different phenomenology, including e.g. three-body decays. Along line 2 we fix $m_{3 / 2}$ such that the resulting squark and gluino masses are around $1.2 \mathrm{TeV}$, so that the benchmark points listed in Table 14 can be probed by upcoming LHC data in the near future. Note however that points along line 2 do not, in general, provide the correct dark matter abundance.

\subsection{Planes, lines and points in the p19MSSM}

In recent fits to a phenomenological MSSM, several points which fitted indirect data well were seen to have the lighter parts of the spectrum rather degenerate [119]. We take inspiration from these points in order to define two simple p19MSSM planes with different phenomenology. We also use the p19MSSM to implement 'simplified models', where one is interested in only a few sparticles giving particular signatures: the rest are set to be heavy and therefore irrelevant at current centre of mass energies. All parameters are defined at the electroweak scale.

\subsection{1 p19MSSM plane I}

$\left(M_{1}, M_{3}=m_{\tilde{f}_{L, R^{*}}}^{1 \text { st } / 2 \text { nd gen }}\right)$ plane:

$M_{2}=m_{\tilde{f}_{L R}}^{\text {3rdgen }}=m_{\tilde{e}_{R}, \tilde{\mu}_{R}}=2500 \mathrm{GeV}$,

$m_{H_{u}}^{2}=m_{H_{d}}^{2}=0, \quad A_{t, b, \tau}=0$,

$\tan \beta=10, \quad \mu>0$

where the * in $m_{\tilde{f}_{R^{*}}}$ implies the exception for $\tilde{e}_{R}$ and $\tilde{\mu}_{R}$ whose masses are fixed. Plane I provides significantly different phenomenology to previous studied MSSM scenarios. For low values of $M_{3}=m_{\tilde{f}_{L, R^{*}}}^{1 \text { st } / 2 \text { ndgen }}$ the plane allows for quasi-degenerate squarks, gluinos and neutralinos, which are likely to result in softer jets and thus be more difficult to detect, for any given mass of squarks and gluinos. For high values of the same parameter the plane captures a light mass gauginos-only scenario that could also be difficult to rule out at a hadron collider. In the latter scenario only neutralino and/or chargino production is possible at the LHC.

\subsection{2 p19MSSM plane II}

$\left(M_{1}, m_{\tilde{l}}\right)$ plane:

$m_{\tilde{e}_{R}}=m_{\tilde{e}_{L}}=m_{\tilde{l}}, \quad \tan \beta=10, \quad \mu>0$,

$M_{3}=M_{2}=m_{\tilde{f}_{L, R}}^{3 \mathrm{rdgen}}=m_{\tilde{u}_{R}, \tilde{d}_{R}, \tilde{q}_{L}}=\mu=M_{A}=2500 \mathrm{GeV}$,

$A_{t, b, \tau}=0$.

We are also interested in models giving di-lepton plus missing transverse momentum signatures, in the absence of hard jets. The simplest model for this is to have light smuons, selectrons and lightest neutralino and everything else heavy. The only SUSY production is therefore neutralino LSP production (possibly resulting in monojet signatures if one includes initial state radiation) or slepton production. One can obtain missing transverse momentum plus zero, one or two leptons.

\subsection{3 p19MSSM lines and points}

We define one p19MSSM line for each p19MSSM parameter plane defined above.

Line p19MSSM1 has $M_{3}=m_{\tilde{f}_{L, R^{*}}}^{1 \text { st } / 2 \text { nd gen }}=1.2 M_{1}$, where $M_{1}$ is an integer multiple of $100 \mathrm{GeV}$. Some points along this line together with their gluino-squark masses and production cross-sections are shown in Table 15. It is currently unknown whether the lighter points have been ruled out by LHC SUSY searches, but a high level of compression in the MSSM spectra is known to drastically reduce the LHC experiments' acceptances, at least with standard cuts [120]. These spectra have been produced with SOFTSUSY3 . 1 . $7[79,80]$.

Line p19MSSM2 is in p19MSSM plane II, defined along a line such that a neutralino is the LSP: $M_{1}=0.75 \times$ $m_{\tilde{l}}, \Delta m_{\tilde{l}}=20 \mathrm{GeV}$. As can be seen from Table 16, the righthanded sleptons are lighter than the left-handed sleptons. All of the points have $100 \%$ branching ratios for a first or second generation charged slepton to go to the same flavour lepton and a neutralino. Sneutrinos may also be produced, resulting in missing transverse momentum, since they decay to neutrinos and neutralinos. 
Table 15 Line p19MSSM1: $M_{3}=m_{\tilde{f}_{L, R^{*}}}^{1 \text { st/2ndgen }}=1.2 M_{1}, \tan \beta=$ $10, \mu>0, M_{2}=m_{\tilde{f}_{L, R}}^{3 \text { rdgen }}=m_{\tilde{e}_{R}, \tilde{\mu}_{R}}=2500 \mathrm{GeV}, m_{H_{u}}^{2}=m_{H_{d}}^{2}=$ $0, A_{t, b, \tau}=0, \Delta M_{1} \stackrel{\nu_{1}}{=} 100 \mathrm{GeV}$ (masses are in units of $\mathrm{GeV}$ and rounded to the nearest $5 \mathrm{GeV}$ ). The cross-section given in the final column is the NLO cross-section of gluino and squark production at $7 \mathrm{TeV}$ proton-proton collider as calculated by prospino [93-95] v2.1

\begin{tabular}{|c|c|c|c|c|c|c|}
\hline Point & $M_{1}$ & $M_{3}=m_{\tilde{f}_{L, R^{*}}}^{1 \text { st } / 2 \text { nd gen }}$ & $m_{\tilde{g}}$ & $\min \left(m_{\tilde{q}}\right)$ & $M_{\tilde{\chi}_{1}^{0}}$ & $\sigma(\mathrm{fb})$ \\
\hline p19MSSM1.1 & 300 & 360 & 435 & 450 & 280 & 30080 \\
\hline p19MSSM1.2 & 400 & 480 & 570 & 525 & 330 & 6123 \\
\hline p19MSSM1.3 & 500 & 600 & 700 & 650 & 340 & 1576 \\
\hline p19MSSM1.4 & 600 & 720 & 830 & 780 & 342 & 467 \\
\hline p19MSSM1.5 & 700 & 840 & 960 & 900 & 345 & 153 \\
\hline p19MSSM1.6 & 800 & 960 & 1090 & 1030 & 345 & 53 \\
\hline p19MSSM1.7 & 900 & 1080 & 1215 & 1150 & 345 & 19 \\
\hline p19MSSM1.8 & 1000 & 1200 & 1340 & 1280 & 345 & 7 \\
\hline p19MSSM1.N & $\ldots$ & $\ldots$ & $\ldots$ & $\ldots$ & $\ldots$ & $\ldots$ \\
\hline
\end{tabular}

Table 16 p19MSSM2: $M_{1}=0.75 \times m_{\tilde{l}}, \tan \beta=10, \mu>0, M_{3}=$ $M_{2}=m_{\tilde{f}_{L, R}}^{3 \mathrm{rd} \text { gen }}=m_{\tilde{u}_{R}, \tilde{d}_{R}}=\mu=M_{A}=2500 \mathrm{GeV}, A_{t, b, \tau}=0, \Delta m_{\tilde{l}}=$ $20 \mathrm{GeV}$ (masses are in units of $\mathrm{GeV}$ and rounded to the nearest
$5 \mathrm{GeV}$ ). The cross-section given in the final column is the cross-section of neutralino and slepton production as calculated by HERWIG++$2.5 .1[116]$

\begin{tabular}{|c|c|c|c|c|c|c|}
\hline Point & $m_{\tilde{l}}$ & $M_{1}$ & $M_{\chi_{1}^{0}}$ & $m_{\tilde{e}_{R}}$ & $m_{\tilde{e}_{L}}$ & $\sigma(\mathrm{fb})$ \\
\hline p19MSSM2.1 & 100 & 75 & 75 & 130 & 240 & 49 \\
\hline p19MSSM2.2 & 120 & 90 & 90 & 150 & 250 & 32 \\
\hline p19MSSM2.3 & 140 & 105 & 100 & 165 & 260 & 28 \\
\hline p19MSSM2.4 & 160 & 120 & 115 & 180 & 270 & 24 \\
\hline p19MSSM2.5 & 180 & 135 & 130 & 200 & 285 & 16 \\
\hline p19MSSM2.6 & 200 & 150 & 145 & 220 & 295 & 14 \\
\hline p19MSSM2.N & $\ldots$ & $\ldots$ & $\ldots$ & $\ldots$ & $\ldots$ & $\ldots$ \\
\hline
\end{tabular}

3.8 Benchmark planes, lines and points in the RPV-CMSSM

To aid comparison with the R-parity conserving case, our RPV planes are based on CMSSM plane I from Sect. 3.2.1, augmented by a single non-zero weak-scale RPV coupling. When scanning over the parameters $m_{0}$ and $m_{1 / 2}$ we propose to include the points at low $m_{0}$ and high $m_{1 / 2}$ where the stau is the LSP, as well as neutralino LSP points. The stau LSP decays have been included in HERWIG [121, 122] in all cases below. For potential analyses, see also the detailed work [123] on looking for stau-LSP scenarios at the LHC.

\subsubsection{RPV-CMSSM plane I}

$\underline{\left(m_{0}, m_{1 / 2}\right) \text { plane: }}$

$\lambda_{121}\left(M_{Z}\right)=0.01, \quad \mu>0, \quad \tan \beta=10, \quad A_{0}=0$.
This plane produces a signal of four charged leptons and also missing $p_{T}$ from two escaping neutrinos along with additional jets etc. from squark decays. SUSY discovery should be easier than the corresponding CMSSM plane due to the presence of the leptons. For low $m_{0}$ and high $m_{1 / 2}$, when the stau becomes the LSP, the stau will decay via a 4-body decay, into three jets and a tau. This is included in HERWIG.

\subsubsection{RPV-CMSSM plane II}

$\left(m_{0}, m_{1 / 2}\right)$ plane:

$\lambda_{112}^{\prime}\left(M_{Z}\right)=0.001, \quad \mu>0$,

$\tan \beta=10, \quad A_{0}=0$.

The neutralino can decay to $e / \nu_{e}+2$ jets. Thus for this coupling the decay of the two neutralinos can lead to two electrons, an electron and a neutrino or two neutrinos in the final state. The two electrons can be opposite sign or same sign, due to the Majorana nature of the neutralino. Furthermore there are two jets from the neutralino decay. The stau LSP 
Table 17 Branching ratios of neutralino LSPs along line RPV1. They are independent of the mass point. Particles marked $v$ may also be antineutrinos

\begin{tabular}{llllll}
\hline Mode & BR & Mode & BR & Mode & BR \\
\hline$\tilde{\chi}_{1}^{0} \rightarrow e^{+} e^{-} v_{\mu}$ & 0.50 & $\tilde{\chi}_{1}^{0} \rightarrow e^{+} \mu^{-} v_{e}$ & 0.25 & $\tilde{\chi}_{1}^{0} \rightarrow e^{-} \mu^{+} v_{e}$ & 0.25 \\
\hline
\end{tabular}

Table 18 Line RPV2: $\lambda_{112}^{\prime}\left(M_{Z}\right)=10^{-3}, m_{0}=50 \mathrm{GeV}, \tan \beta=10$, $A_{0}=0, \mu>0, \overline{\Delta m_{1 / 2}}=50 \mathrm{GeV}$ (masses in $\mathrm{GeV}$, rounded to $5 \mathrm{GeV}$ accuracy, branching ratios in \%). Particles marked $v$ may also be

\begin{tabular}{lllllllll}
\hline Point & $m_{1 / 2}$ & $m_{\tilde{\chi}_{1}^{0}}$ & $m_{\tilde{\tau}_{1}}$ & $m_{\tilde{g}}$ & $\left\langle m_{\tilde{q}}\right\rangle$ & $c \tau(\mathrm{mm})$ & $\mathrm{BR}(\tilde{\tau} \rightarrow \tau j j v)$ & $\mathrm{BR}(\tilde{\tau} \rightarrow e j j \tau)$ \\
\hline RPV2.1 & 400 & 160 & 155 & 935 & 840 & $8 \times\left(10^{-3} / \lambda_{112}^{\prime}\right)^{2}$ & 60 & 40 \\
RPV2.2 & 450 & 185 & 175 & 1040 & 935 & $10 \times\left(10^{-3} / \lambda_{112}^{\prime}\right)^{2}$ & 57 & 41 \\
RPV2.3 & 500 & 205 & 190 & 1150 & 1030 & $11 \times\left(10^{-3} / \lambda_{112}^{\prime}\right)^{2}$ & 57 & 43 \\
RPV2.4 & 550 & 225 & 210 & 1250 & 1120 & $11 \times\left(10^{-3} / \lambda_{112}^{\prime}\right)^{2}$ & 56 & 4 \\
RPV2.N & $\ldots$ & $\ldots$ & $\ldots$ & $\ldots$ & $\ldots$ & $\ldots$ & $\ldots$ & $\ldots$ \\
\hline
\end{tabular}

anti-neutrinos, and in the final column, the $e$ denotes electrons and positrons. The charge of the $\tau$ is identical to that of the $\tilde{\tau}$ region has a four-body decay leading to two additional $\tau \mathrm{s}$ in the final state.

\subsubsection{RPV-CMSSM plane III}

$\left(m_{0}, m_{1 / 2}\right)$ plane:

$\lambda_{123}^{\prime \prime}\left(M_{Z}\right)=1 \times 10^{-4}, \quad \tan \beta=10, \quad A_{0}=0$.

Here, we have the minimal inclusive eight-jet signature with no missing transverse momentum. The search strategies proposed so far rely on the charged leptons from the cascade decay [124, 125] and the possible jet structure [126].

\subsubsection{Lines}

Each RPV-CMSSM parameter plane has one associated line in parameter space. For two of the parameter planes, we simply use CMSSM Line 10.1, whereas for the other, we choose a line with a stau LSP to illustrate scenarios with additional taus in the final-state topology. We leave the RPV parameter $\Lambda$ unchanged.

Line RPV1 This line is defined as $\lambda_{121}\left(M_{Z}\right)=0.01, m_{0}=$ $0.25 \times m_{1 / 2}$, with reference points spaced in steps of $\Delta m_{1 / 2}=50 \mathrm{GeV}$. Using the di-leptons from cascade decays involving $\tilde{\chi}_{2}^{0}$ may be complicated by the presence of electrons and muons coming from the LSP decay. The inclusive signal of this line is 2 jets plus four leptons, each of which may be neutral, a muon or an electron. The muon and electron can be of either charge. Thus possible combinations of four charged leptons are $e^{+} e^{+} e^{-} e^{-}, e^{+} e^{-} \mu^{+} e^{-}$, $e^{+} e^{-} \mu^{-} e^{+}, \mu^{+} \mu^{+} e^{-} e^{-}, \mu^{-} \mu^{-} e^{+} e^{+}, \mu^{+} \mu^{-} e^{+} e^{-}$, with the fourth and fifth particularly spectacular. The basic features of the spectra of points RPV1.1-RPV1.5 are given in Table 2, with neutralino decays as in Table 17.
Table 19 Branching ratios of neutralino LSPs along line RPV3. They are independent of the mass point

\begin{tabular}{llll}
\hline Mode & Branching ratio & Mode & Branching ratio \\
\hline$\tilde{\chi}_{1}^{0} \rightarrow j j b$ & 0.50 & $\tilde{\chi}_{1}^{0} \rightarrow j j \bar{b}$ & 0.50 \\
\hline
\end{tabular}

Line RPV2 $\lambda_{112}^{\prime}\left(M_{Z}\right)=10^{-3}, m_{0}=50 \mathrm{GeV}$, with reference points spaced in steps of $\Delta m_{1 / 2}=50 \mathrm{GeV}$. This line has a neutralino LSP for $m_{1 / 2}<350 \mathrm{GeV}$, but a stau LSP in the selected range of reference points $m_{1 / 2} \geq 400 \mathrm{GeV}$. For the chosen value of $\lambda_{112}^{\prime}\left(M_{Z}\right)=10^{-3}$, the stau decay length is in the range of $1 \mathrm{~cm}$, as shown in Table 18. The final state signature, when the staus decay inside the detector, is six jets plus two first-generation leptons plus two taus. The charged leptons can be like-sign or opposite sign.

Line RPV3 $\quad \lambda_{123}^{\prime \prime}\left(M_{Z}\right)=1 \times 10^{-4}, m_{0}=0.25 \times m_{1 / 2}$, with reference points spaced in steps of $\Delta m_{1 / 2}=50 \mathrm{GeV}$. The inclusive signal of this model is 6 jets, $2 b$-jets and no intrinsic missing transverse momentum. The additional leptons coming from cascades involving $\tilde{\chi}_{2}^{0}$ will likely provide a useful handle when searching for this difficult SUSY scenario. The basic features of the spectra of points RPV3.1-RPV3.5 are given in Table 2, with neutralino decays as in Table 19.

\subsection{Benchmark lines and points in the NMSSM}

As stated in Sect. 2.9, $m_{0}$ and $\lambda$ are required to be small in the cNMSSM, leaving $m_{1 / 2}$ as the only essential free parameter. Fixing $m_{0}=0$ and $\lambda=10^{-3}$ defines a Line $\mathbf{c N}$ MSSM with points listed in Table 20. More properties of these points (obtained from the NMHDECAY/NMSPEC/ NMSDECAY computer codes [127-130]) and proposed cuts can be found in [131]. In the cNMSSM the LSP is 
always singlino-like and the NLSP is a stau with slightly larger mass. This configuration leads to sparticle decay cascades that are typically rich in $\tau$-leptons.

The fact that there is also motivation for nonuniversal boundary conditions (vs. the complete universality in the cNMSSM), and the fact that $\lambda$ and $m_{0}$ must be so small in the cNMSSM in order to have correct phenomenology suggests that it may be important to consider the slightly less restricted scenarios of the CNMSSM and the SNMSSM as described earlier.

A global study of the CNMSSM [88] has shown that (preLHC) data strongly "pull" $\lambda$ and $\kappa$ towards zero, which is a decoupling limit. In this limit the phenomenology of the model very much resembles that of the CMSSM. Nevertheless, both $\lambda$ and $\kappa$ are allowed to be substantially different from zero, with $\kappa \sim \lambda$. The lightest neutralino tends to be bino dominated, and the lightest Higgs scalar is SM-like, just as in the CMSSM. For this reason we believe that the benchmark lines and planes defined above for the CMSSM are applicable also to the CNMSSM. On the flip side of the coin, in the preferred region of parameter space it is likely to be very challenging to distinguish between the two models at the LHC. Additional work is needed to clarify this in more detail.

The sNMSSM, on the other hand, leads to a vastly expanded range of scenarios. One scenario that exemplifies this is outlined below. We give only a single point SNMSSM1.1 specified by the parameters given in Table 21; more will be added in the future.

Some of the interesting and, indeed, intriguing aspects of this point are as follows. The LSP has a mass of $\sim 21 \mathrm{GeV}$ and is primarily singlino-like with correct relic density from s-channel annihilation through the singlet-like Higgs boson, $h_{1}$, where $m_{h_{1}} \simeq 49 \mathrm{GeV}$. The SM-like Higgs, $h_{2}$, has $m_{h_{2}} \simeq 115 \mathrm{GeV}$. The lightest CP-odd Higgs boson is singlet in nature and has mass $m_{a_{1}} \sim 21 \mathrm{GeV}$. The lightest stop has

Table 20 Line cNMSSM: $m_{0}=0$ and $\lambda=10^{-3}$. (Masses in GeV; gluino and squark masses rounded to $5 \mathrm{GeV}$ accuracy)

\begin{tabular}{lccccc}
\hline Point & $m_{1 / 2}$ & $m_{\tilde{g}}$ & $m_{\tilde{u}_{R}}$ & $m_{\tilde{\chi}_{1}^{0}}$ & $m_{\tilde{\tau}_{1}}$ \\
\hline cNMSSM.1 & 520 & 1190 & 1045 & 142 & 147 \\
cNMSSM.2 & 600 & 1360 & 1190 & 166 & 171 \\
cNMSSM.3 & 800 & 1780 & 1545 & 225 & 229 \\
cNMSSM.4 & 1000 & 2190 & 1895 & 282 & 286 \\
cNMSSM.N & $\ldots$ & $\ldots$ & $\ldots$ & $\ldots$ & $\ldots$ \\
\hline
\end{tabular}

a mass of $\sim 504 \mathrm{GeV}$, but all other squarks and the gluino have masses above a $\mathrm{TeV}$.

Decay chains are quite exotic. For example, squarks and gluinos often decay down to the $\tilde{\chi}_{2}^{+}$, the latter decaying $37 \%$ of the time into $\tilde{\chi}_{1}^{+} h_{2}$ and $20 \%$ of the time to $\tilde{\chi}_{1}^{+} Z$. The $h_{2}$ decays $68 \%$ of the time to the invisible singlinos and $20 \%$ of the time into $h_{1} h_{1}$, where the $h_{1}$ decays $74 \%$ of the time to $a_{1} a_{1}$, with $a_{1}$ decaying $92 \%$ of the time to $b \bar{b}$. As a result, $15 \%$ of all $h_{2}$ decays and $2 \%$ of all left-handed squark decays result in a final state containing eight $b$ quarks.

This point serves to illustrate the huge range of scenarios that are possible in the context of the SNMSSM. It will be quite challenging to discover such a scenario. The same applies to many other "exotic" scenarios that are possible within the SNMSSM on the basis of limits on either Higgs or supersymmetric particles.

\section{Summary}

We have proposed in this document various benchmark scenarios for future SUSY searches at the LHC. These benchmark subspaces of the MSSM could be useful for the presentation of experimental results, and the specific benchmark points illustrate different possible experimental signatures for testing and comparing detector performances. In making our proposals, we have been guided by the impressive reaches already demonstrated by the LHC experiments, which already exclude large domains of the parameter spaces of some well-studied models. On the other hand, even larger domains of parameter space remain to be explored with the increases in luminosity and centre-of-mass energy currently envisaged. We include in our selection of benchmarks parameter planes that have already been partially explored as well as embedded lines and discrete points, some of which have already been explored by the LHC and other experiments but may serve as useful points for comparison. The points are defined on parameter lines such that they can be extended to higher masses. Thus as one point becomes excluded, a new point is already defined (the lightest one not ruled out by an LHC experiment to $95 \%$ confidence level).

Our most detailed proposals have been for the CMSSM and related models, but we have also made proposals in the contexts of alternative scenarios for supersymmetry breaking. These proposals are less developed and detailed than in the CMSSM, reflecting the fact that, historically, the phenomenologies of these models have been studied less.
Table 21 Point sNMSSM1.1: GUT scale parameters (masses in $\mathrm{GeV}$ ) except for $\tan \beta$ and $\lambda$ which are given at scale $m_{Z}$

\begin{tabular}{llllllllll}
\hline$\lambda$ & $m_{1 / 2}$ & $m_{0}$ & $m_{H_{u}}$ & $m_{H_{d}}$ & $A_{0}$ & $A_{\kappa}$ & $A_{\lambda}$ & $\tan \beta$ & $\operatorname{sign}(\mu)$ \\
\hline 0.406 & 481 & 1078 & 5639 & 868 & -1500 & -664 & -1870 & 3.13 & - \\
\hline
\end{tabular}


In view of the increasing pressure applied by the LHC to the CMSSM, such alternative models may merit more phenomenological attention in the future. In order to encourage this, we also provide explicit proposals for benchmark points and parameter planes in the mGMSB, mAMSB, and the $R_{p}$-violating CMSSM. We also include some simplified models with specific collider topologies by exploiting a 19dimensional parameterisation of a phenomenologically parameterised weak-scale MSSM, the p19MSSM.

We note that many of the supersymmetric signatures (for example jets plus missing transverse momentum) are insensitive to the presence of Higgs scalars, as long as their couplings are not too large so as to change the supersymmetric spectrum. Thus, some features of our planes, lines and points may apply also to the NMSSM, as well as to MSSMbased models. However, it is likely that Higgs signals appearing in cascade decays would be different in the NMSSM and singlino LSPs could extend the length of the cascades. Further, if the LHC should discover a SM-like Higgs above about $140 \mathrm{GeV}$, which is above the upper limit of the MSSM but not the NMSSM, the focus of SUSY searches would obviously shift away from the MSSM to non-minimal realisations of SUSY. The same would apply should a relatively light Higgs boson be observed decaying to a pair of still lighter scalars. We have included proposals for benchmark points and a parameter line in the NMSSM, in order to illustrate the potentially more "exotic" phenomenology in this model.

\begin{abstract}
Acknowledgements We thank C. Lester, F. Moortgat, L. Pape, P. Pralavorio, G. Redlinger, D. Stuart and A. Tapper for their input and feedback to the proposals presented here. M.K. would like to thank J. Conley, L. Glaser and J. Tattersall for helpful discussions on MMASMB scenarios. H.K.D. would like to thank Tim Stefaniak and Klaus Desch for helpful discussions. The work of J.E. and K.A.O. was supported partly by the London Centre for Terauniverse Studies (LCTS), using funding from the European Research Council via the Advanced Investigator Grant 267352. U.E. acknowledges support from the French ANR LFV-CPV-LHC. J.F.G. is supported by U.S. DOE grant No. DE-FG03-91ER40674. The work of S.H. was supported in part by CICYT (grant FPA 2010-22163-C02-01) and by the Spanish MICINN's Consolider-Ingenio 2010 Program under grant MultiDark CSD2009-00064. The work of K.A.O. is supported in part by DOE grant DE-FG02-94ER-40823 at the University of Minnesota.
\end{abstract}

Open Access This article is distributed under the terms of the Creative Commons Attribution Noncommercial License which permits any noncommercial use, distribution, and reproduction in any medium, provided the original author(s) and source are credited.

\section{References}

1. H.P. Nilles, Phys. Rep. 110, 1 (1984)

2. H.E. Haber, G.L. Kane, Phys. Rep. 117, 75 (1985)

3. R. Barbieri, Riv. Nuovo Cimento 11, 1 (1988)

4. S.P. Martin, arXiv:hep-ph/9709356

5. G. Aad et al. (ATLAS Collaboration), arXiv:1107.0561 [hep-ex]
6. G. Aad et al. (ATLAS Collaboration), Eur. Phys. J. C 71, 1682 (2011). arXiv:1103.6214 [hep-ex]

7. G. Aad et al. (ATLAS Collaboration), Eur. Phys. J. C 71, 1647 (2011). arXiv:1103.6208 [hep-ex]

8. G. Aad et al. (ATLAS Collaboration), Phys. Lett. B 701, 398 (2011). arXiv:1103.4344 [hep-ex]

9. G. Aad et al. (ATLAS Collaboration), Phys. Lett. B 701, 1 (2011). arXiv:1103.1984 [hep-ex]

10. J.B.G. da Costa et al. (ATLAS Collaboration), Phys. Lett. B 701, 186 (2011). arXiv:1102.5290 [hep-ex]

11. G. Aad et al. (ATLAS Collaboration), Phys. Rev. Lett. 106, 131802 (2011). arXiv:1102.2357 [hep-ex]

12. S. Chatrchyan et al. (CMS Collaboration), CERN-PH-EP-2011138

13. S. Chatrchyan et al. (CMS Collaboration), J. High Energy Phys. 1108, 156 (2011). arXiv:1107.1870 [hep-ex]

14. S. Chatrchyan et al. (CMS Collaboration), arXiv:1107.1279 [hep-ex]

15. S. Chatrchyan et al. (CMS Collaboration), J. High Energy Phys. 1108, 155 (2011). arXiv:1106.4503 [hep-ex]

16. S. Chatrchyan et al. (CMS Collaboration), J. High Energy Phys. 1107, 113 (2011). arXiv:1106.3272 [hep-ex]

17. S. Chatrchyan et al. (CMS Collaboration), arXiv:1106.0933 [hep-ex]

18. S. Chatrchyan et al. (CMS Collaboration), J. High Energy Phys. 1106, 093 (2011). arXiv:1105.3152 [hep-ex]

19. S. Chatrchyan et al. (CMS Collaboration), J. High Energy Phys. 1106, 077 (2011). arXiv:1104.3168 [hep-ex]

20. S. Chatrchyan et al. (CMS Collaboration), J. High Energy Phys. 1106, 026 (2011). arXiv:1103.1348 [hep-ex]

21. S. Chatrchyan et al. (CMS Collaboration), Phys. Rev. Lett. 106, 211802 (2011). arXiv:1103.0953 [hep-ex]

22. V. Khachatryan et al. (CMS Collaboration), Phys. Lett. B 698, 196 (2011). arXiv:1101.1628 [hep-ex]

23. V. Khachatryan et al. (CMS Collaboration), Phys. Rev. Lett. 106, 011801 (2011). arXiv:1011.5861 [hep-ex]

24. D. Alves, N. Arkani-Hamed, S. Arora, Y. Bai, M. Baumgart, J. Berger, M. Buckley, B. Butler et al., arXiv:1105.2838 [hep-ph]

25. G.L. Kane, C.F. Kolda, L. Roszkowski, J.D. Wells, Phys. Rev. D 49, 6173 (1994). arXiv:hep-ph/9312272

26. H. Baer, M. Brhlik, Phys. Rev. D 53, 597 (1996). arXiv: hep-ph/9508321

27. H. Baer, M. Brhlik, Phys. Rev. D 57, 567 (1998). arXiv: hep-ph/9706509

28. J.R. Ellis, T. Falk, K.A. Olive, M. Schmitt, Phys. Lett. B 388, 97 (1996). arXiv:hep-ph/9607292

29. J.R. Ellis, T. Falk, K.A. Olive, M. Schmitt, Phys. Lett. B 413, 355 (1997). arXiv:hep-ph/9705444

30. J.R. Ellis, T. Falk, G. Ganis, K.A. Olive, M. Schmitt, Phys. Rev. D 58, 095002 (1998). arXiv:hep-ph/9801445

31. V.D. Barger, C. Kao, Phys. Rev. D 57, 3131 (1998). arXiv: hep-ph/9704403

32. J.R. Ellis, T. Falk, G. Ganis, K.A. Olive, Phys. Rev. D 62, 075010 (2000). arXiv:hep-ph/0004169

33. J.R. Ellis, T. Falk, G. Ganis, K.A. Olive, M. Srednicki, Phys. Lett. B 510, 236 (2001). arXiv:hep-ph/0102098

34. V.D. Barger, C. Kao, Phys. Lett. B 518, 117 (2001). arXiv: hep-ph/0106189

35. L. Roszkowski, R. Ruiz de Austri, T. Nihei, J. High Energy Phys. 0108, 024 (2001). arXiv:hep-ph/0106334

36. A. Djouadi, M. Drees, J.L. Kneur, J. High Energy Phys. 0108, 055 (2001). arXiv:hep-ph/0107316

37. U. Chattopadhyay, A. Corsetti, P. Nath, Phys. Rev. D 66, 035003 (2002). arXiv:hep-ph/0201001

38. J.R. Ellis, K.A. Olive, Y. Santoso, New J. Phys. 4, 32 (2002). arXiv:hep-ph/0202110 
39. H. Baer, C. Balazs, A. Belyaev, J.K. Mizukoshi, X. Tata, Y. Wang, J. High Energy Phys. 0207, 050 (2002). arXiv:hep-ph/0205325

40. R. Arnowitt, B. Dutta, arXiv:hep-ph/0211417

41. O. Buchmueller et al., Eur. Phys. J. C 71, 1583 (2011). arXiv:1011.6118 [hep-ph]

42. J. Polonyi, Hungary Central Inst Res-KFKI-77-93

43. E. Cremmer, B. Julia, J. Scherk, P. van Nieuwenhuizen, S. Ferrara, L. Girardello, Phys. Lett. B 79, 231 (1978)

44. E. Cremmer, B. Julia, J. Scherk, S. Ferrara, L. Girardello, P. van Nieuwenhuizen, Nucl. Phys. B 147, 105 (1979)

45. R. Barbieri, S. Ferrara, C.A. Savoy, Phys. Lett. B 119, 343 (1982)

46. A.H. Chamseddine, R.L. Arnowitt, P. Nath, Phys. Rev. Lett. 49, 970 (1982)

47. J.R. Ellis, K.A. Olive, Y. Santoso, V.C. Spanos, Phys. Lett. B 573, 162 (2003). arXiv:hep-ph/0305212

48. J.R. Ellis, K.A. Olive, Y. Santoso, V.C. Spanos, Phys. Rev. D 70, 055005 (2004). arXiv:hep-ph/0405110

49. H. Baer, A. Mustafayev, S. Profumo, A. Belyaev, X. Tata, Phys. Rev. D 71, 095008 (2005). arXiv:hep-ph/0412059

50. H. Baer, A. Mustafayev, S. Profumo, A. Belyaev, X. Tata, J. High Energy Phys. 0507, 065 (2005). hep-ph/0504001

51. J.R. Ellis, K.A. Olive, P. Sandick, Phys. Rev. D 78, 075012 (2008). arXiv:0805.2343 [hep-ph]

52. D. Matalliotakis, H.P. Nilles, Nucl. Phys. B 435, 115-128 (1995). hep-ph/9407251

53. V. Berezinsky, A. Bottino, J.R. Ellis, N. Fornengo, G. Mignola, S. Scopel, Astropart. Phys. 5, 1-26 (1996). hep-ph/9508249

54. J.R. Ellis, K.A. Olive, Y. Santoso, Phys. Lett. B 539, 107 (2002). arXiv:hep-ph/0204192

55. J.R. Ellis, T. Falk, K.A. Olive, Y. Santoso, Nucl. Phys. B 652, 259 (2003). arXiv:hep-ph/0210205

56. L. Roszkowski, R. Ruiz de Austri, R. Trotta, Y.L. Tsai, T.A. Varley, Phys. Rev. D 83, 015014 (2011). [Phys. Rev. D 83, 039901 (2011)]. arXiv:0903.1279 [hep-ph]

57. L.E. Ibanez, G.G. Ross, Phys. Lett. B 110, 215 (1982)

58. G.F. Giudice, R. Rattazzi, Phys. Rep. 322, 419-499 (1999). hep-ph/9801271

59. L. Randall, R. Sundrum, Nucl. Phys. B 557, 79-118 (1999). hep-th/9810155

60. G.F. Giudice, M.A. Luty, H. Murayama, R. Rattazzi, J. High Energy Phys. 9812, 027 (1998). hep-ph/9810442

61. S. Ambrosanio, G.D. Kribs, S.P. Martin, Phys. Rev. D 56, 17611777 (1997). hep-ph/9703211

62. B.C. Allanach, M.J. Dolan, arXiv:1107.2856 [hep-ph]

63. J.R. Ellis, K.A. Olive, P. Sandick, Phys. Lett. B 642, 389 (2006). arXiv:hep-ph/0607002

64. J.R. Ellis, K.A. Olive, P. Sandick, J. High Energy Phys. 0706, 079 (2007). arXiv:0704.3446 [hep-ph]

65. J.R. Ellis, K.A. Olive, P. Sandick, J. High Energy Phys. 0808, 013 (2008). arXiv:0801.1651 [hep-ph]

66. S. Kachru, R. Kallosh, A.D. Linde, S.P. Trivedi, Phys. Rev. D 68, 046005 (2003). arXiv:hep-th/0301240

67. K. Choi, A. Falkowski, H.P. Nilles, M. Olechowski, Nucl. Phys. B 718, 113-133 (2005). hep-th/0503216

68. H. Baer, E.K. Park, X. Tata, T.T. Wang, J. High Energy Phys. 0608, 041 (2006). arXiv:hep-ph/0604253

69. A. Djouadi, J.L. Kneur, G. Moultaka, Comput. Phys. Commun. 176, 426 (2007). arXiv:hep-ph/0211331. Version 2.3 of the code was used

70. S.S. AbdusSalam, AIP Conf. Proc. 1078, 297-299 (2009). arXiv:0809.0284 [hep-ph]

71. C.F. Berger, J.S. Gainer, J.L. Hewett, T.G. Rizzo, J. High Energy Phys. 0902, 023 (2009). arXiv:0812.0980 [hep-ph]

72. S.S. AbdusSalam, B.C. Allanach, F. Quevedo, F. Feroz, M. Hobson, Phys. Rev. D 81, 095012 (2010). arXiv:0904.2548 [hep-ph]
73. A. Fowlie, L. Roszkowski, arXiv:1106.5117 [hep-ph]

74. J.A. Conley, J.S. Gainer, J.L. Hewett, M.P. Le, T.G. Rizzo, arXiv:1103.1697 [hep-ph]

75. S.S. AbdusSalam, F. Quevedo, Phys. Lett. B 700, 343-350 (2011). arXiv:1009.4308 [hep-ph]

76. N.E. Bomark, S. Lola, P. Osland, A.R. Raklev, Phys. Lett. B 677, 62 (2009). arXiv:0811.2969 [hep-ph]

77. B.C. Allanach, A. Dedes, H.K. Dreiner, Phys. Rev. D 69, 115002 (2004). hep-ph/0309196

78. M. Hirsch, J.W.F. Valle, W. Porod, J.C. Romao, A. Villanova del Moral, Phys. Rev. D 78, 013006 (2008). arXiv:0804.4072 [hep-ph]

79. B.C. Allanach, M.A. Bernhardt, Comput. Phys. Commun. 181, 232-245 (2010). arXiv:0903.1805 [hep-ph]

80. B.C. Allanach, Comput. Phys. Commun. 143, 305-331 (2002). hep-ph/0104145

81. H.P. Nilles, M. Srednicki, D. Wyler, Phys. Lett. B 120, 346 (1983)

82. J.R. Ellis, J.F. Gunion, H.E. Haber, L. Roszkowski, F. Zwirner, Phys. Rev. D 39, 844 (1989)

83. M. Drees, Int. J. Mod. Phys. A 4, 3635 (1989)

84. U. Ellwanger, C. Hugonie, A.M. Teixeira, Phys. Rep. 496, 1 (2010)

85. J.F. Gunion, D.E. Lopez-Fogliani, L. Roszkowski, R.R. de Austri, T.A. Varley, arXiv:1105.1195 [hep-ph]

86. A. Djouadi, U. Ellwanger, A.M. Teixeira, Phys. Rev. Lett. 101, $101802(2008)$

87. A. Djouadi, U. Ellwanger, A.M. Teixeira, J. High Energy Phys. 0904, 031 (2009)

88. D.E. Lopez-Fogliani, L. Roszkowski, R.R. de Austri, T.A. Varley, Phys. Rev. D 80, 095013 (2009). arXiv:0906.4911 [hep-ph]

89. B.C. Allanach, M. Battaglia, G.A. Blair, M.S. Carena, A. De Roeck, A. Dede, A. Djouadi, D. Gerdes et al., Eur. Phys. J. C 25, 113-123 (2002). hep-ph/0202233

90. P.Z. Skands, B.C. Allanach, H. Baer, C. Balazs, G. Belanger, F. Boudjema, A. Djouadi, R. Godbole et al., J. High Energy Phys. 0407, 036 (2004). hep-ph/0311123

91. M.J. Dolan, D. Grellscheid, J. Jaeckel, V.V. Khoze, P. Richardson, J. High Energy Phys. 1106, 095 (2011). arXiv:1104.0585 [hep-ph]

92. ATLAS Collaboration, ATLAS-CONF-2011-086 (2011)

93. W. Beenakker, R. Höpker, M. Spira, P.M. Zerwas, Nucl. Phys. B 492, 51-103 (1997). hep-ph/9610490

94. W. Beenakker, M. Krämer, T. Plehn, M. Spira, P.M. Zerwas, Nucl. Phys. B 515, 3-14 (1998). hep-ph/9710451

95. W. Beenakker, M. Klasen, M. Krämer, T. Plehn, M. Spira, P.M. Zerwas, Phys. Rev. Lett. 83, 3780-3783 (1999). hep-ph/9906298

96. B.C. Allanach, Phys. Rev. D 83, 095019 (2011). arXiv:1102. 3149 [hep-ph]

97. P. Bechtle, B. Sarrazin, K. Desch, H.K. Dreiner, P. Wienemann, M. Krämer, C. Robens, B. O’Leary, Phys. Rev. D 84, 011701 (2011). arXiv:1102.4693 [hep-ph]

98. O. Buchmueller, R. Cavanaugh, D. Colling, A. de Roeck, M.J. Dolan, J.R. Ellis, H. Flacher, S. Heinemeyer et al., Eur. Phys. J. C 71, 1634 (2011). arXiv:1102.4585 [hep-ph]

99. R. Trotta, F. Feroz, M.P. Hobson, L. Roszkowski, R. Ruiz de Austri, J. High Energy Phys. 0812, 024 (2008). arXiv:0809.3792 [hep-ph]

100. B.C. Allanach, T.J. Khoo, C.G. Lester, S.L. Williams, J. High Energy Phys. 1106, 035 (2011). arXiv:1103.0969 [hep-ph]

101. G. Bertone, D.G. Cerdeno, M. Fornasa, R.R. de Austri, C. Strege, R. Trotta, arXiv:1107.1715 [hep-ph]

102. P. Bechtle, K. Desch, H. Dreiner, M. Kramer, B. O'Leary, C. Robens, B. Sarrazin, P. Wienemann, arXiv:1105.5398 [hep-ph]

103. O. Buchmueller, R. Cavanaugh, D. Colling, A. De Roeck, M.J. Dolan, J.R. Ellis, H. Flacher, S. Heinemeyer et al., Eur. Phys. J. C 71, 1722 (2011). arXiv:1106.2529 [hep-ph] 
104. A. Strumia, J. High Energy Phys. 1104, 073 (2011). arXiv:1101.2195 [hep-ph]

105. D. Feldman, K. Freese, P. Nath, B.D. Nelson, G. Peim, Phys. Rev. D 84, 015007 (2011). arXiv:1102.2548 [hep-ph]

106. S. Scopel, S. Choi, N. Fornengo, A. Bottino, Phys. Rev. D 83, 095016 (2011). arXiv:1102.4033 [hep-ph]

107. S. Akula, N. Chen, D. Feldman, M. Liu, Z. Liu, P. Nath, G. Peim, Phys. Lett. B 699, 377-382 (2011). arXiv:1103.1197 [hep-ph]

108. S. Akula, D. Feldman, Z. Liu, P. Nath, G. Peim, Phys. Lett. B 699, 377-381 (2011). arXiv:1103.5061 [hep-ph]

109. A. Strumia, arXiv:1107.1259 [hep-ph]

110. M. Farina, M. Kadastik, D. Pappadopulo, J. Pata, M. Raidal, A. Strumia, arXiv:1104.3572 [hep-ph]

111. O. Buchmueller et al., http://mastercode.web.cern.ch/mastercode/ index.php. arXiv: 1110.3568 [hep-ph]

112. A. Fowlie, A. Kalinowski, M. Kazana, L. Roszkowski, Y.L.S. Tsai (BayesFITS Collaboration), arXiv:1111.6098 [hep-ph]

113. A. De Roeck, J.R. Ellis, F. Gianotti, F. Moortgat, K.A. Olive, L. Pape, Eur. Phys. J. C 49, 1041 (2007). arXiv:hep-ph/0508198. This is an update of benchmark point B originally proposed in M. Battaglia et al., Eur. Phys. J. C 22, 535 (2001). arXiv: hep-ph/0106204

114. M. Battaglia, A. De Roeck, J.R. Ellis, F. Gianotti, K.A. Olive, L. Pape, Eur. Phys. J. C 33, 273 (2004). arXiv:hep-ph/0306219

115. M. Muhlleitner, A. Djouadi, Y. Mambrini, Comput. Phys. Commun. 168, 46 (2005). arXiv:hep-ph/0311167. Version 1.3 of the code was used

116. M. Bahr et al., Eur. Phys. J. C 58, 639 (2008). arXiv:0803.0883 [hep-ph]

117. F.E. Paige, S.D. Protopopescu, H. Baer, X. Tata, hep-ph/ 0312045
118. J.A. Conley, H.K. Dreiner, L. Glaser, M. Krämer, J. Tattersall, arXiv: 1110.1287 [hep-ph]

119. S.S. AbdusSalam, arXiv:1106.2317 [hep-ph]

120. T.J. LeCompte, S.P. Martin, Phys. Rev. D 84, 015004 (2011). arXiv:1105.4304 [hep-ph]

121. G. Corcella, I.G. Knowles, G. Marchesini, S. Moretti, K. Odagiri, P. Richardson, M.H. Seymour, B.R. Webber, J. High Energy Phys. 0101, 010 (2001). HERWIG 6.5, hep-ph/0011363

122. G. Corcella, I.G. Knowles, G. Marchesini, S. Moretti, K. Odagiri, P. Richardson, M.H. Seymour, B.R. Webber, hep-ph/0210213

123. K. Desch, S. Fleischmann, P. Wienemann, H.K. Dreiner, S. Grab, Phys. Rev. D 83, 015013 (2011). arXiv:1008.1580 [hep-ph]

124. H. Baer, C. Kao, X. Tata, Phys. Rev. D 51, 2180-2186 (1995). hep-ph/9410283

125. B.C. Allanach, A.J. Barr, M.A. Parker, P. Richardson, B.R. Webber, J. High Energy Phys. 0109, 021 (2001). hep-ph/0106304

126. J.M. Butterworth, J.R. Ellis, A.R. Raklev, G.P. Salam, Phys. Rev. Lett. 103, 241803 (2009). arXiv:0906.0728 [hep-ph]

127. U. Ellwanger, J.F. Gunion, C. Hugonie, J. High Energy Phys. 0502, 066 (2005)

128. U. Ellwanger, C. Hugonie, Comput. Phys. Commun. 175, 290 (2006)

129. U. Ellwanger, C. Hugonie, Comput. Phys. Commun. 177, 399 (2007)

130. D. Das, U. Ellwanger, A.M. Teixeira, arXiv:1106.5633 [hep-ph]

131. U. Ellwanger, A. Florent, D. Zerwas, J. High Energy Phys. 1101, $103(2011)$ 